\title{
Tristetraprolin expression by keratinocytes controls local and systemic inflammation
}

\author{
Mathieu Andrianne, ${ }^{1}$ Assiya Assabban, ${ }^{1}$ Caroline La, ${ }^{1}$ Denis Mogilenko, ${ }^{2}$ \\ Delphine Staumont Salle, ${ }^{2}$ Sébastien Fleury, ${ }^{2}$ Gilles Doumont, ${ }^{3}$ Gaëtan Van Simaeys, ${ }^{3,4}$ \\ Sergei A. Nedospasov, ${ }^{5}$ Perry J. Blackshear, ${ }^{6,7}$ David Dombrowicz, ${ }^{2}$ Stanislas Goriely, ${ }^{1}$ \\ and Laurye Van Maele ${ }^{1}$ \\ 'Walloon Excellence in Lifesciences and Biotechnology (WELBIO) and Institute for Medical Immunology, Université Libre \\ de Bruxelles (ULB), Brussels, Belgium. ${ }^{2}$ Université de Lille, Inserm, Institut Pasteur de Lille, CHU Lille, Lille, France. ${ }^{3}$ Centre \\ of Microscopy and Molecular Imaging (CMMI), ULB, Charleroi (Gosselies), Belgium. ${ }^{4}$ Department of Nuclear Medicine, \\ Hôpital Erasme, ULB, Brussels, Belgium. ${ }^{5}$ Engelhardt Institute of Molecular Biology, Russian Academy of Sciences \\ and Lomonosov Moscow State University, Moscow, Russia. ${ }^{6}$ Signal Transduction Laboratory, National Institute of \\ Environmental Health Sciences, Research Triangle Park, North Carolina, USA. ${ }^{7}$ Departments of Medicine and Biochemistry, \\ Duke University Medical Center, Durham, North Carolina, USA.
}

Tristetraprolin (TTP, encoded by the Zfp36 gene) regulates the mRNA stability of several important cytokines. Due to the critical role of this RNA-binding protein in the control of inflammation, TTP deficiency leads to the spontaneous development of a complex inflammatory syndrome. So far, this phenotype has been largely attributed to dysregulated production of TNF and IL-23 by myeloid cells, such as macrophages or DCs. Here, we generated mice with conditional deletion of TTP in keratinocytes (Zfp36 $6^{\mathrm{fl} / \mathrm{fI}} \mathrm{K} 14-C r e$ mice, referred to herein as Zfp36 $6^{\mathrm{EP}}$ mice). Unlike DC-restricted (CD11c-Cre) or myeloid cell-restricted (LysM-Cre) TTP ablation, these mice developed exacerbated inflammation in the imiquimod-induced psoriasis model. Furthermore, Zfp $36^{\Lambda \mathrm{EP}}$ mice progressively developed a spontaneous pathology with systemic inflammation, psoriatic-like skin lesions, and dactylitis. Finally, we provide evidence that keratinocyte-derived TNF production drives these different pathological features. In summary, these findings expand current views on the initiation of psoriasis and related arthritis by revealing the keratinocyte-intrinsic role of TTP.

Authorship note: M. Andrianne and A Assabban are co-first authors. S. Goriely and L. Van Maele are cosenior authors.

Conflict of interest: The authors have declared that no conflict of interest exists.

Submitted: January 23, 2017

Accepted: May 3, 2017

Published: June 2, 2017

\section{Reference information:}

JCI Insight. 2017;2(11):e92979.

https://doi.org/10.1172/jci.

insight. 92979.

\section{Introduction}

Psoriasis is a chronic disease with a complex etiology that affects skin and joints in $1 \%-3 \%$ of the general population. It is characterized by inflamed and scaly skin lesions and oligoarthritis of the small joints of the hands and feet, and it arises in genetically susceptible hosts in response to ill-defined environmental triggers (1). While psoriasis was previously considered as an autoimmune disease, the current pathogenic model emphasizes the central role of innate immune populations, including plasmacytoid and inflammatory myeloid DCs (2). Dysregulated production of TNF, IL-23, and other inflammatory cytokines was shown to promote IL-17 and IL-22 expression by multiple lymphoid subpopulations. In response to these STAT3-activating cytokines, keratinocytes proliferate and produce chemokines and cytokines, such as IL-36 family members, that then contribute to a positive feedback loop of inflammation. Both TNF and IL-23/IL-17 axes therefore represent major therapeutic targets that have dramatically changed the management of psoriasis and psoriatic arthritis (3).

Despite these major advances, there remain important knowledge gaps impeding a full understanding of psoriasis pathophysiology. Indeed, the initial triggers that set this vicious circle in motion in genetically predisposed individuals are still poorly defined. Release of cytokines and danger signals, such as nucleic acids, by stressed or dying keratinocytes could be important initiating factors (4). While cutaneous symptoms generally precede the onset of psoriatic arthritis, the pathophysiological link between skin and joint lesions also remains elusive (5). Psoriatic arthritis is clinically and genetically related to the spondylarthropathies. IL-23 is thought to play a major role in these pathologies, as systemic IL-23 expression was shown to activate ROR $\gamma \mathrm{t}^{+}$entheseal resident $\mathrm{T}$ cells, leading to local inflammation and enthesitis (6). In the context of inflammatory bowel diseases, it was therefore suggested that gut-derived IL-23 induces distant joint inflammation (7). Whether a similar process may occur during skin inflammation remains unknown. 
Multiple mechanisms may control the duration or intensity of inflammatory processes. It is now widely recognized that, in addition to transcriptional regulation, expression of some key proinflammatory genes is controlled at the posttranscriptional level by the degradation of their mRNA. The presence of AU-rich elements (AREs) in their 3' untranslated regions allows the recruitment of RNA-binding proteins (such as tristetraprolin [TTP], encoded by the Zfp36 gene) and subsequent RNA destabilization. In macrophages or DCs, TTP acts as a major regulator of RNA degradation and controls the production of key inflammatory cytokines, such as TNF, IL-1 $\beta$, IL-6, or IL-23 $(8,9)$. In line with these observations, TTP-deficient mice spontaneously develop a complex TNF- and IL-23-dependent inflammatory syndrome characterized by cachexia, progressive dermatitis, arthritis, and myeloid hyperplasia $(10,11)$. However, ablation of TTP within the myeloid compartment $\left(Z f p 36^{\mathrm{H} / \mathrm{fl}}\right.$ LysMCre mice, referred to herein as $Z f p 36^{\mathrm{MM}}$ mice) did not lead to spontaneous inflammation, despite increased sensitivity to endotoxin shock $(8,12)$. Hence, the pathophysiological processes that lead to the spontaneous development of this syndrome remain poorly defined.

Herein, we explored the role of TTP in the imiquimod-induced psoriasis model. These experiments uncover a critical keratinocyte-intrinsic role of TTP in the control of local skin inflammation. Furthermore, deficiency of TTP in keratinocytes resulted in the progressive development of a spontaneous pathology with systemic inflammation, skin lesions, and dactylitis. Finally, we show that absence of TNF production by keratinocytes abolishes these pathological features.

\section{Results}

Exacerbation of imiquimod-induced skin inflammation in $Z f p 36^{-1-}$ mice. In comparison to the severe phenotype initially described by Taylor et al. (10), $Z f p 36^{-1-}$ mice bred under strict specific pathogen-free conditions rarely developed any signs of skin or joint inflammation before the age of 8 to 10 weeks. Younger mice (5-6 weeks) displayed reduced weight gain but were otherwise found to be globally healthy and could therefore be used in short-term experiments. To investigate the mechanisms involved in skin pathology associated with TTP deficiency, we used the classical model of imiquimod-induced psoriasis-like inflammation (topical application over 5 days), in which IL-23 and IL-17 play an important role. We observed that $Z f p 36^{-1}$ mice developed a more severe skin pathology than their WT littermates $\left(Z f p 36^{+/+}\right)$, as they displayed increased desquamation, redness, and epidermal thickening (Figure 1A). This was associated with an increased proportion of Ki67 $7^{+}$ basal keratinocytes (Figure 1A) and a massive infiltration of neutrophils and, to a lesser extent, of monocytes, in $Z f p 36^{-/-}$mice as compared with their WT counterparts (Figure 1B). Consistent with the fact that these mice will develop a spontaneous IL-23/IL-17-dependent skin pathology (11), the frequency of IL-17A-expressing cells in mock-treated $Z f p 36^{-1}$ mice was increased as compared with control WT mice. Despite this observation, the proportion of skin-infiltrating IL-17A-producing cells was not significantly upregulated in imiquimod-treated $Z f p 36^{--}$mice, but we observed an induction of cells producing both IL-17A and IL-22 in this group. As previously reported (13), these cytokine-producing cells were predominantly $\gamma \delta \mathrm{T}$ cells, although conventional $\alpha \beta \mathrm{T}$ cells and innate lymphoid cells also contributed (Figure 1C and gating strategy in Supplemental Figure 1; supplemental material available online with this article; https://doi.org/10.1172/jci. insight.92979DS1). We found elevated levels of some inflammatory genes (such as CXCl2, Il17a, and Il23a) in the skin of mock-treated $Z f p 36^{-1-}$ mice, suggestive of low-grade spontaneous inflammation (Figure 1D). As expected, imiquimod treatment strongly enhanced expression of psoriasis-associated inflammatory mediators and revealed elevated levels of cytokines (such as Il23a, Il22, Il19), chemokines (Cxcl2), and antimicrobial peptides (S100a8, S100a9) in the skin of $Z f p 36^{-1-}$ mice (Figure 1E). Taken together, these results indicate that, in line with the known role of TTP in the posttranscriptional control of key inflammatory cytokines, this RNA-binding protein represents an important regulator of local skin inflammation.

Central role of TTP expression by keratinocytes, rather than myeloid cells, in the control of imiquimod-induced skin inflammation. So far, the physiological role of TTP has been mainly assessed in myeloid cells, such as macrophages or DCs. Furthermore, DCs are known to play a central role in the imiquimod-induced psoriasis model. In order to address the role of TTP within immune versus nonimmune cells, we performed bone marrow chimera experiments. For this purpose, lethally irradiated congenic CD45.1 (WT) or CD45.2 $Z f p 36^{-1-}$ mice were injected with bone marrow cells from either source. Eight weeks after reconstitution, mice were subjected to imiquimod treatment. We observed strong exacerbation of the skin phenotype, along with increased neutrophils and IL-17A-producing cell infiltration when recipient mice were deficient for TTP (Supplemental Figure 2, A-C). In contrast, WT mice reconstituted with TTP-deficient bone marrow cells did not display increased sensitivity to imiquimod treatment. These experiments suggest that TTP 
A

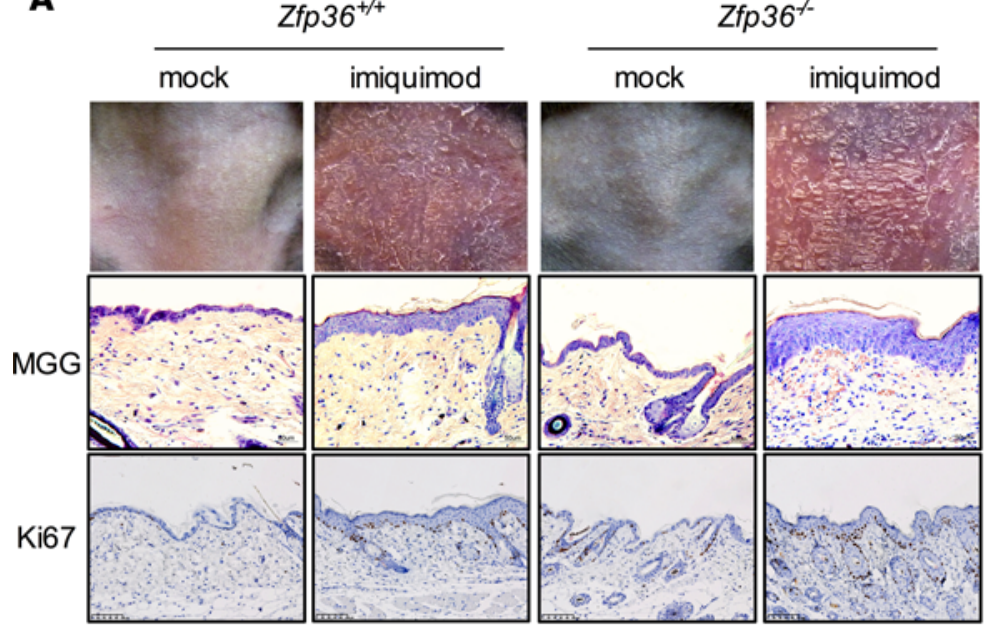

B

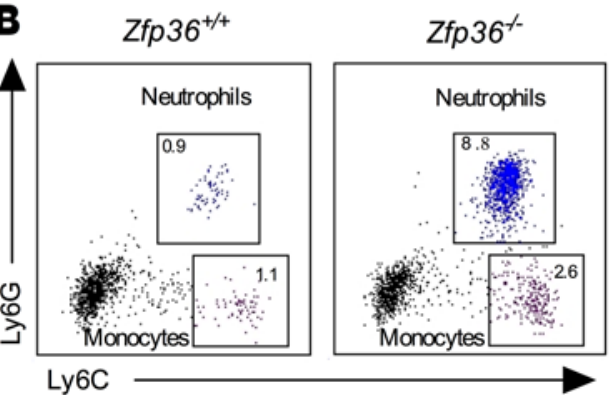

C

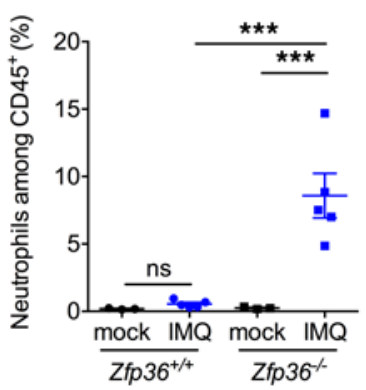

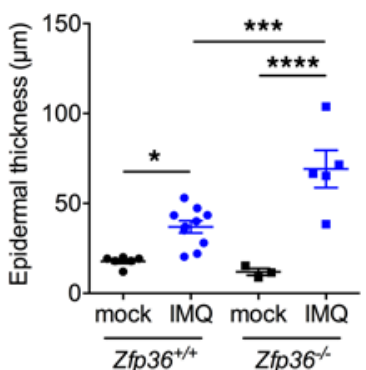
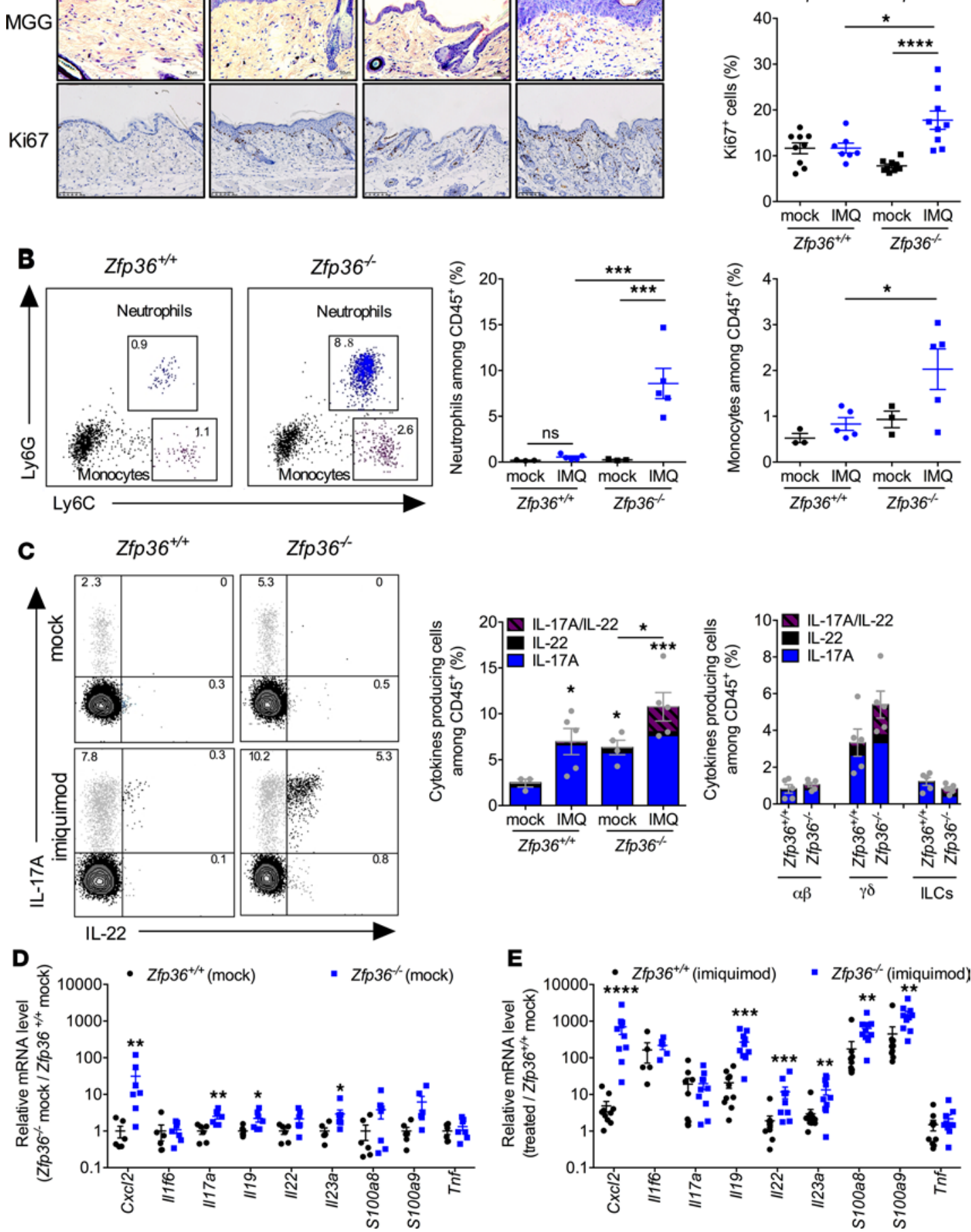

Figure 1. Loss of tristetraprolin increases the severity of imiquimod-induced skin lesions. Zfp36-deficient mice (Zfp36 $\left.6^{-/-}\right)$and their littermates $\left(Z f p 36^{+/+}\right)$were treated topically with imiquimod over 5 consecutive days $(n=5)$ or not $(n=3)$. Skin samples were collected 4 hours after the last application for analysis of epidermal thickness by histology (A), cell recruitment by flow cytometry (B), cytokine expression by intracellular protein staining (C), or transcript levels by RTqPCR ( $\mathbf{D}$ and E). (A) Representative macroscopic images of the abdomen via MGG staining of skin sections (original magni- 
fication, $\times 200$ ) from 13 mock Zfp36 $6^{+/+}, 21$ imiquimod-treated $Z f p 36^{+/+}, 13$ mock Zfp36-/, and 22 imiquimod-treated Zfp36 (labeling index, $n=3$ by group); and epidermal thickness. (B) Representative FACS plots and manual gating strategy for neutrophil and monocyte

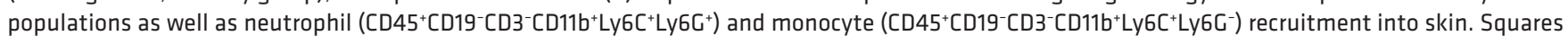
represent gates for neutrophil and monocyte populations. (C) IL-17A and IL-22 production by CD45+CD19-NK1.1-Gr1- cells and cytokine production by

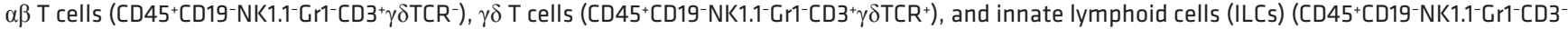
CD90 $\left.+C D 127^{+}\right)$. Each dot represents total cytokine production in an individual mouse. (D and E) Total skin mRNA levels in mock-treated animals (D) $(n=$ 7 mice by group, pooled from 2 experiments) and in imiquimod-treated animals (E) ( $n=10$ mice by group, pooled from 2 experiments) were normalized against Actb, Gapdh, and Hprt mRNA levels and expressed relative to the Zfp36 $6^{+/+}$mock group arbitrarily set to 1 . Bars represent mean \pm SEM. Statistical significance $\left({ }^{*} P<0.05\right.$, ${ }^{* *} P<0.01,{ }^{* *} P<0.001$, $\left.{ }^{* * *} P<0.0001\right)$ was assessed by 1-way ANOVA test with Bonferroni correction (A-C) or 2-tailed Mann-Whitney compared with mock-treated $Z f p 36^{+/+}$group (D) or compared with imiquimod-treated Zfp $36^{+/+}$group (E). Results are representative of 5 independent experiments. Results observed with ear or dorsum were presented in Supplemental Figure 1A (see also Supplemental Figure 1).

within radioresistant cells or nonreplaceable cells (either from the stroma or from hematopoietic origin) rather than sensitive cells from the hematopoietic system contributed to the phenotype of $Z f p 36^{-/}$mice. However, because of the experimental design, interpretation of these results is difficult, as some mice developed signs of spontaneous skin and joint inflammation. We therefore generated mice with cell-specific deletion of TTP in the DC compartment $\left(Z f p 36^{\mathrm{G} / \mathrm{f}} \mathrm{CD} 11 \mathrm{cCre}\right.$ mice, referred to herein as $Z f p 36^{\mathrm{ADC}}$ mice) These mice did not display a higher susceptibility to imiquimod treatment (Figure 2), despite increased sensitivity to endotoxin shock with strongly elevated circulating TNF and IL-23 levels, an observation reminiscent of myeloid-restricted TTP deletion (ref. 12 and Supplemental Figure 3). Similar results were obtained in the imiquimod model with these mice ( $Z f p 36^{\triangle \mathrm{M}}$ mice), indicating that TTP expression by DCs, monocytes/macrophages, or granulocytes does not play a major role in this inducible dermatitis model.

It was recently reported that keratinocytes express TTP protein (14). We therefore generated mice with conditional deletion of TTP in keratinocytes ( $Z f p 36^{6 / 1 / 1} \mathrm{~K} 14-C r e$ mice, referred to herein as $Z f p 36^{1 \mathrm{EP}}$ mice). The use of this K14Cre strain was shown to efficiently and specifically mediate excision in epidermal CD45-Ep$\mathrm{CAM}^{+}$cells (15). We observed a strong reduction of Zfp36 levels in skin samples from these mice (Supplemental Figure 3A), indicating that keratinocytes represent a major cell population expressing TTP in the skin. Strikingly, these mice developed severe inflammation upon imiquimod treatment to a similar extent as whole-body Zfp36-deficient mice (Figure 2A and Supplemental Figure 3C). This exacerbated phenotype was associated with increased recruitment of neutrophils and IL-17A-producing $\gamma \delta \mathrm{T}$ cells and $\alpha \beta \mathrm{T}$ cells but not monocytes in skin lesions (Figure 2, B-D). Next, we analyzed expression of inflammatory signature genes in mock- and imiquimod-treated skin samples of $Z f p 36^{\mathrm{EP}}$ and $Z f p 36^{\natural / f}$ control mice (Figure 2, E and F). The expression pattern in $Z f p 36^{\mathrm{AEP}}$ mice was globally comparable to the one observed in $Z f p 36^{-/}$mice (Figure $1, \mathrm{D}$ and E). Of note, Zfp36 expression was reduced by $60 \%-80 \%$ in $Z f p 36^{\triangle \mathrm{EP}}$ mice and was found to be slightly increased upon imiquimod treatment in $Z f p 36^{\sharp / f}$ mice. To further evaluate the molecular mechanisms underlying the exacerbation of psoriasis in $Z f p 36^{\mathrm{AEP}}$ mice, we performed transcriptomic analysis of total skin upon imiquimod treatment. As expected, numerous transcripts were significantly $(q<0.05)$ dysregulated in response to imiquimod treatment in skin from $Z f p 36^{\sharp / f}$ mice, including 1,828 significantly upregulated and 1,377 significantly downregulated genes (Figure 3A). Among increased transcripts, TTP deficiency significantly affected the expression levels of known genes associated with skin inflammation and abnormal keratinocyte differentiation, such as S100a8/9, Defb3, Lce3a, and multiple Sprr genes. Although dysregulated genes were present among both increased and suppressed transcripts, the gene set induced by imiquimod (1,828 genes) was globally upregulated in skin lesions from $Z f p 36^{\mathrm{AE}}$ mice as compared with $Z f p 36^{1 / f t}$ mice (Figure $\left.3 \mathrm{~A}\right)$. In contrast, the gene set suppressed by imiquimod (1,377 genes) was clearly skewed toward downregulated genes and was significantly repressed in skin from imiquimod-treated $Z f p 36^{\triangle \mathrm{EP}}$ mice as compared with $Z f p 36^{\prime / f l}$ mice (Figure $3 \mathrm{~A}$ ). Genes that were affected by imiquimod and significantly dysregulated $(q<0.05)$ in the skin from imiquimod-treated $Z f p 36^{\triangle \mathrm{EP}}$ mice compared with $Z f p$ $36^{A / f}$ mice are depicted in Figure 3B. Using gene set enrichment analysis, we highlighted the 4 most significantly $(P<0.001)$ enriched KEGG pathways in skin from imiquimod-treated $Z f p 36^{\mathrm{AEP}}$ mice as compared with $Z f p 36^{A \prime}$ ${ }^{f}$ mice (Figure $3 C$ ). Interestingly, they included proinflammatory signaling pathways, such as epithelial cell signaling in helicobacter infection, cytosolic DNA sensing, NOD-like receptor signaling, and cytokine-cytokine receptor interaction pathways. We focused on the later pathway in more detail. Analysis of the core enrichment genes revealed that many cytokines and chemokines increased in $Z f p 36^{\mathrm{APP}}$ skin but were moderately regulated by imiquimod in skin from $Z f p 36^{\sharp / f}$ mice (Figure 3, D and E). These data indicated that $Z f p 36$ deficiency in keratinocytes resulted in a significant increase of multiple mRNAs encoding cytokines and chemokines that can exacerbate psoriasis, such as II23a, Il12b, Tnf, Illb, Il17a, and II22. 


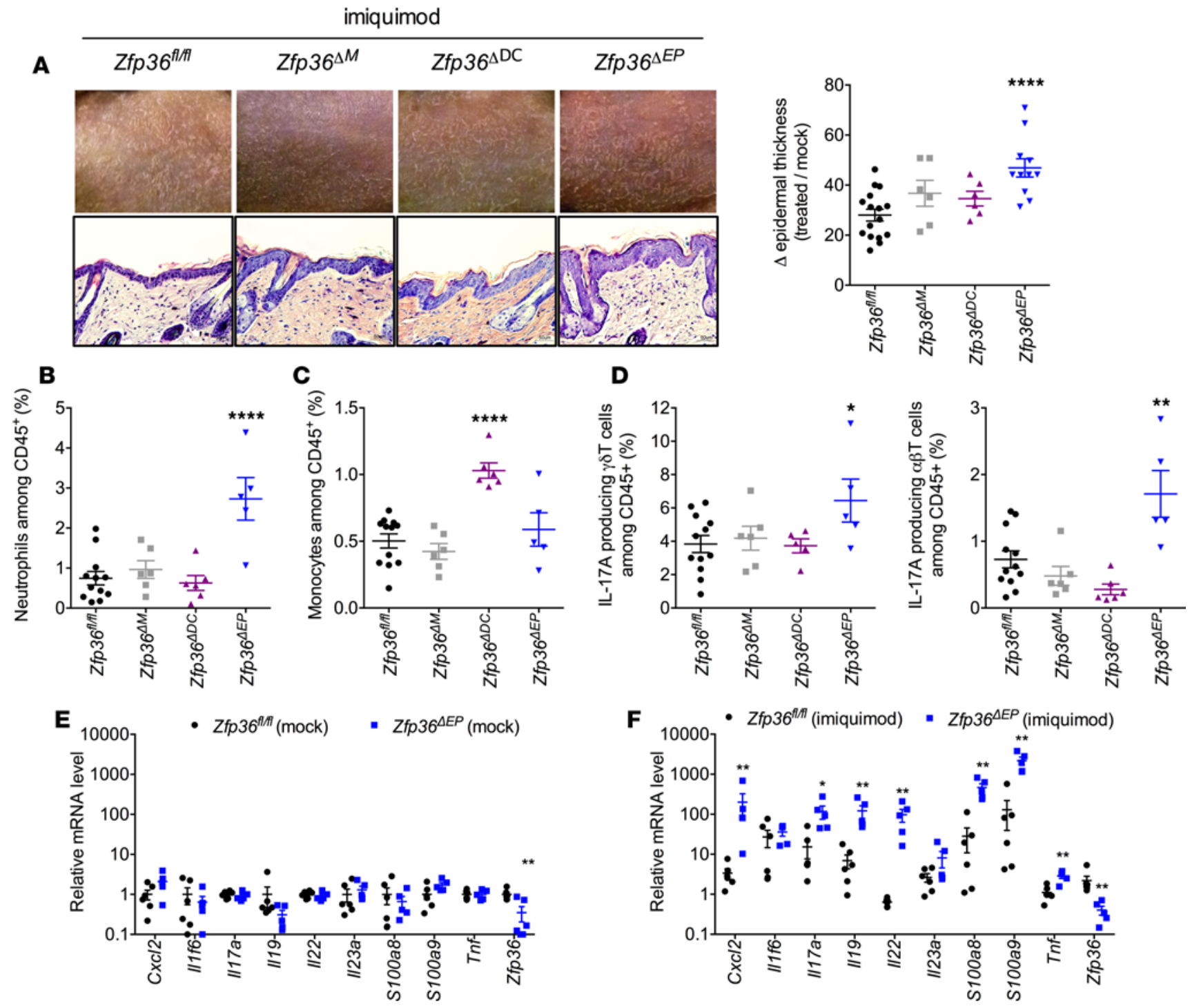

Figure 2. TTP expression by keratinocytes is important for the control of skin inflammation induced by imiquimod. (A-E) Myeloid-specific Zfp36-deficient mice (Zfp36 ${ }^{\triangle M}, n=6$ ), DC-specific Zfp36-deficient mice (Zfp36 $\left.{ }^{\triangle D C}, n=6\right)$, keratinocyte-specific Zfp36-deficient mice $\left(Z f p 36^{\triangle E P}, n=5\right)$, and their littermate controls ( $Z f p 36^{f / f l}, n=12$ ) were topically treated over 5 consecutive days with imiquimod. Skin samples were collected 4 hours after the last application for analysis of epidermal thickness by histology. Original magnification: $\times 200$. (A), cell recruitment by flow cytometry (B and C), cytokine production by intracellular protein staining (D), or transcript levels by RTqPCR (E and $\mathbf{F}$ ). Results are given as mean \pm SEM. Total skin mRNA levels were normalized against Actb, Gapdh, and Hprt mRNA levels and expressed relative to the Zfp $36^{f / f l}$ mock group arbitrarily set to 1 . Statistical significance $\left({ }^{*} P<0.05\right.$, ${ }^{* *} P<0.01$, ${ }^{* * * *} P<0.0001$ ) was assessed by 1-way ANOVA test with Bonferroni correction compared with the Zfp36 f//fl mock group (A-D) or 2-tailed Mann-Whitney test compared with the mock-treated $Z f p 36^{f / f l}$ group $(\mathbf{E})$ or compared with the imiquimod-treated $Z f p 36^{f / f l}$ group (F). Results are representative of at least 5 experiments. Histology is representative of $29 \mathrm{Zfp} 36^{f / f l}, 6 \mathrm{Zfp} 36^{\triangle M}, 18 \mathrm{Zfp} 36^{\triangle D C}$, and $24 \mathrm{Zfp} 36^{\triangle E P}$ mice, pooled from 4 experiments. Representative histology for the mock group from each genotype is presented in Supplemental Figure 3 (see also Supplemental Figure 2).

Skin transcriptome of imiquimod-treated $Z f p 36^{1 E P}$ mice displays important features of human psoriasis. In order to assess the relevance of these findings to human psoriasis, we performed microarray analyses of human laser-microdissected epidermis from affected skin of patients with psoriasis and healthy skin from subjects with no diagnosed skin pathologies. We identified 6,004 genes significantly upregulated and 6,900 genes significantly downregulated in human psoriatic epidermis (Benjamini-Hochberg-adjusted $P<0.05$ ) (Figure $4 \mathrm{~A}$ ). Next, we tested these psoriasis-associated gene sets for differential expression in $Z f p 36^{\mathrm{AEP}}$ mice compared with $Z f p 36^{A / f l}$ mice, both treated with imiquimod. Importantly, the gene set increased in human psoriatic epidermis was significantly upregulated in skin from $Z f p 36^{\Delta \mathrm{EP}}$ mice (Figure $4 \mathrm{~A}$ ). At the same time, the gene set negatively associated with human psoriasis was significantly suppressed in skin from imiquimod-treated $Z f p 36^{\triangle \mathrm{EP}}$ mice (Figure $4 \mathrm{~A}$ ). The genes that are associated with human psoriatic epidermis and 
A

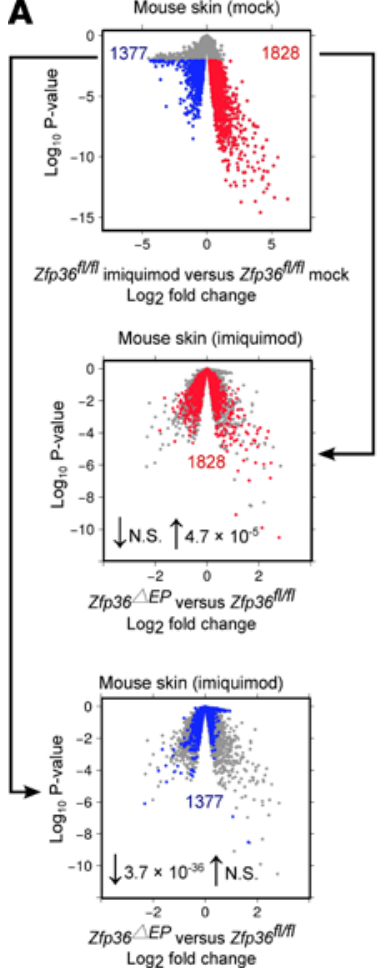

B

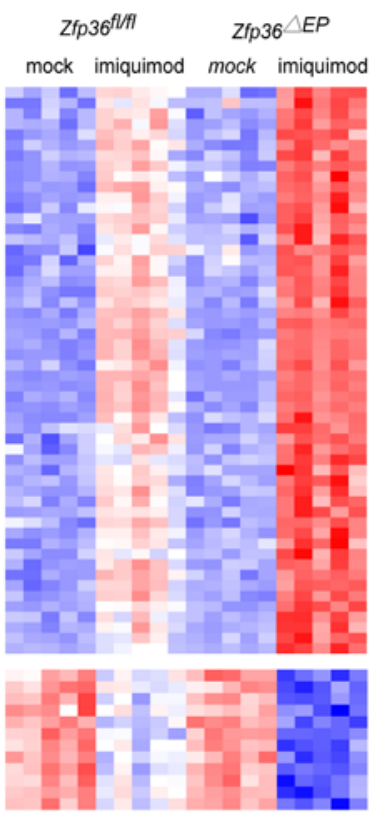

Relative expression

Down-regulated Up-regulated
C

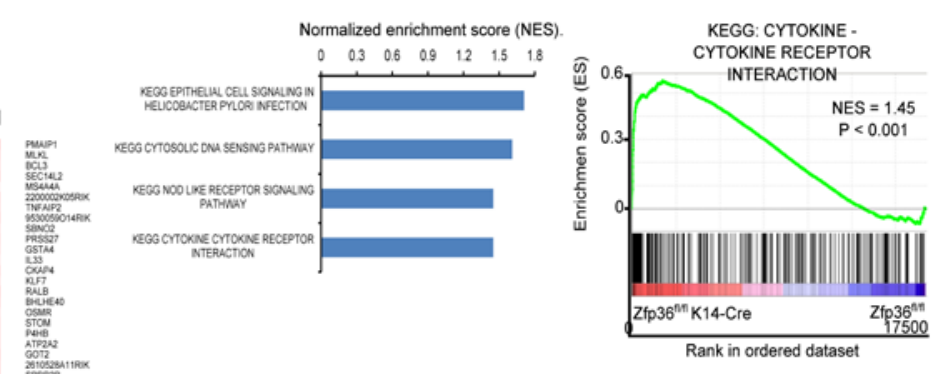

$\mathbf{E}$

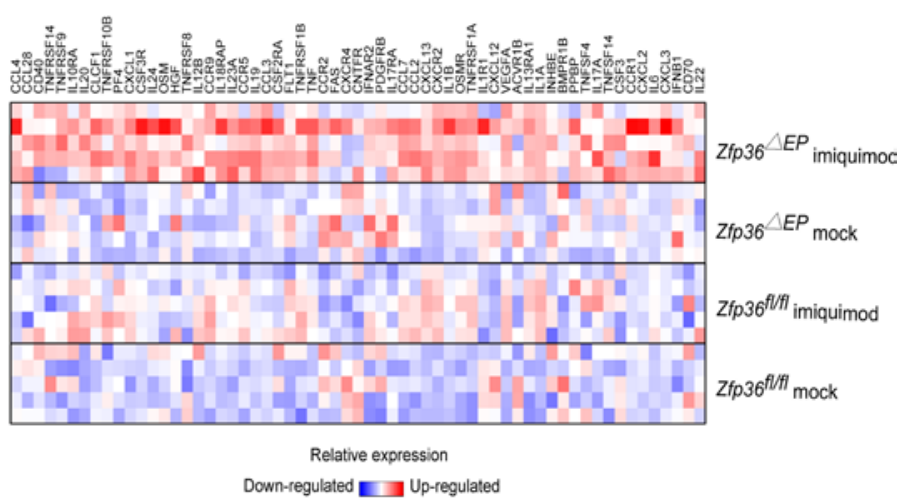

Figure 3. Zfp36 deficiency in keratinocytes results in an increase of multiple transcripts encoding proinflammatory cytokines and chemokines. Zfp $36^{\triangle E P}$ mice and their littermate controls $\left(Z f p 36^{f / f}\right)(n=5)$ were topically treated over 5 consecutive days with imiquimod. Skin samples were collected 4 hours after the last application for transcriptomic analysis. (A) Volcano plot showing $\log _{2}$ fold changes versus $\log _{10} P$ values. As shown from the top of $\mathbf{A}$ to the bottom, 1,828 transcripts were significantly upregulated (red) and 1,377 transcripts were significantly downregulated (blue) among all transcripts (gray) in skin from imiquimod-treated $Z f p 36^{f / f l}$ mice compared with mock-treated $Z f p 36^{f / f l}$ mice. Distribution of the 1,828 transcripts (red, the positive imiquimod signature) among all transcripts (gray) in skin from $Z f p 36^{\triangle E P}$ mice compared with $Z f p 36^{f / f}$ mice, both treated by imiquimod, and $P$ values for differential expression (upregulation or downregulation) of the gene set (red) are shown. The same analysis was performed for the 1,377 transcripts (blue, the negative imiquimod signature). (B) Heatmap of expression levels of transcripts from the positive and negative imiquimod signatures (as shown in A) significantly $(q<0.05$ ) increased or decreased, respectively, in skin from imiquimod-treated $Z f p 36^{\Lambda E P}$ mice compared with imiquimod-treated $Z f p 36^{f / f l}$ mice. (C) Gene set enrichment analysis of 4 of the most significantly $(P<0.001)$ enriched KEGG pathways in skin from imiquimod-treated Zfp36 $6^{4 E P}$ mice compared with Zfp36 $6^{f / f l}$ mice. (D) Enrichment score distribution for the KEGG cytokine-cytokine receptor interaction pathway. $(P<0.001$, permutation test) (E) Heatmap of expression levels of genes from the core enrichment of the KEGG cytokine-cytokine receptor interaction pathway. Each column corresponds to a separate mouse.

that are significantly $(q<0.05)$ dysregulated in skin from $Z f p 36^{\triangle \mathrm{EP}}$ mice are depicted in Figure $4 \mathrm{~A}$. Interestingly, many identified genes involved in human psoriasis, such as IL1B, ARG1, SOD2, SAA2, KRT77, CKB, and others, showed minor, if any, changes in skin from $Z f p 36^{\mathrm{B} / \mathrm{fl}}$ mice treated with imiquimod (Figure 4B). Thus, the skin transcriptome of imiquimod-treated $Z f p 36^{\mathrm{EPP}}$ mice showed important features of human psoriasis, which were mild or absent in WT mice. Notably, in these samples, we observed reduced ZFP36 expression in psoriatic patients as compared with healthy individuals (Figure 4C). These results suggest that the physiological function of TTP might be compromised in the context of human psoriasis.

$T N F$ production by keratinocytes mediates the exacerbation of imiquimod-induced inflammation in Zfp36 $1 E P$ mice. In order to define the functional role of TTP in keratinocytes, we cultured primary keratinocytes isolated from newborn $Z f p 36^{\mathrm{AEP}}$ mice or their littermates. As expected, $Z f p 36^{\mathrm{AEP}}$ cells present a reduction of $Z f p 36 \mathrm{mRNA}$ levels and TTP protein. In control cells, we observed a marked upregulation of $Z f p 36 \mathrm{mRNA}$ levels in response to TPA, a PKC agonist. (Figure 5A). TPA treatment upregulated the expression of cytokine and chemokine genes, such as Tnf, Cxcl1, Cxcl2, Cxcl10, Il19, or Illf6. Induction of Tnf and Cxcl2 was further increased in TTP-deficient keratinocytes (Figure 5B). Heightened expression of Tnf and Cxcl2 was also observed upon stimulation of TTP-deficient cells with rIL-17 or rIL-22, two cytokines that may act on keratinocytes in the context of psoriasis (Figure 5, C and D).

To assess the role of TTP in the control of these cytokines in keratinocytes, we analyzed the halflives of these mRNAs in the presence of actinomycin D. Although p38 MAPK is required for TTP expression and TTP protein stability, it simultaneously blocks the mRNA-destabilizing activity of TTP 
A

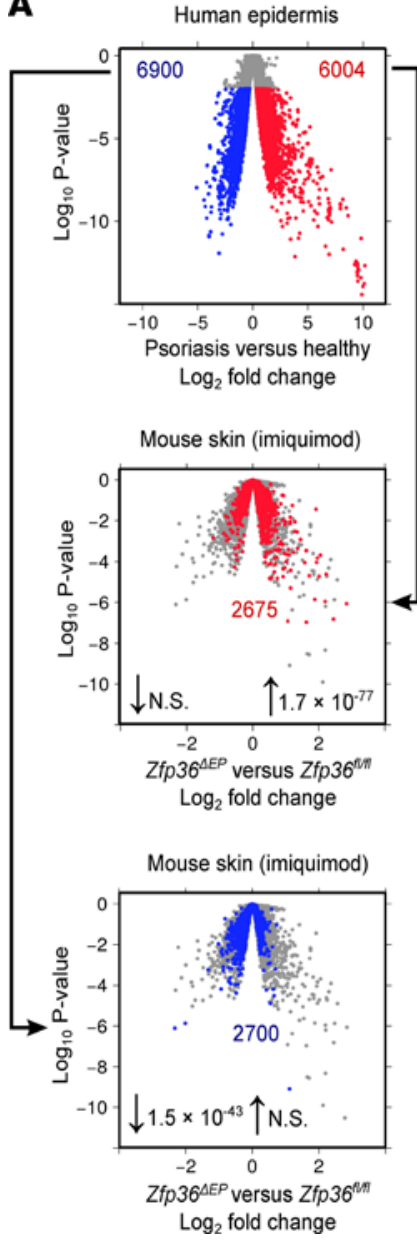

B

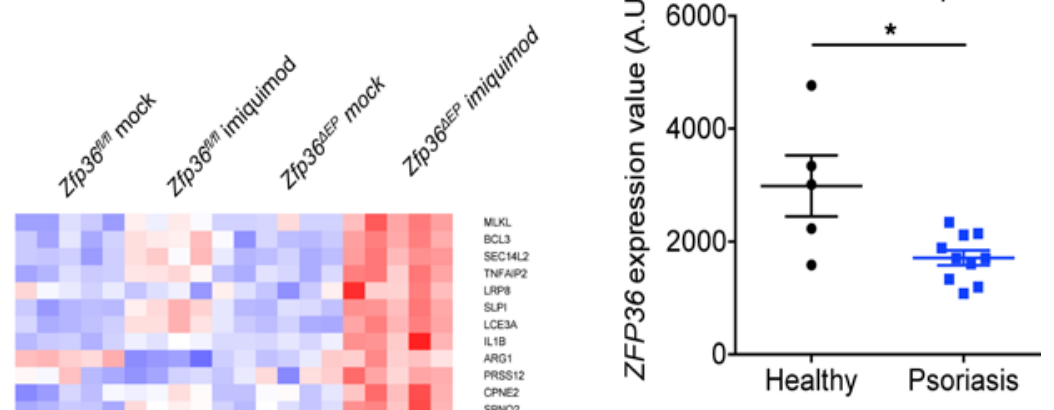

Figure 4. Mice with Zfp36 deficiency in keratinocytes represent a more specific model of human psoriasis. (A) Volcano plot showing log fold $_{2}$ changes versus $\log _{10} P$ values. As shown from the top of $\mathbf{A}$ to the bottom, 6,004 transcripts were significantly upregulated (red) and 6,900 transcripts were significantly downregulated (blue) among all transcripts (gray) in laser-microdissected epidermis from skin of patients with psoriasis compared with healthy epidermis from skin from subjects with no diagnosed skin diseases. Distribution of 2,675 mouse orthologs corresponding to the 6,004 human transcripts increased in psoriatic epidermis (red, the positive psoriasis signature) among all genes (gray) in skin from Zfp $36^{4 E P}$ mice compared with Zfp36 ${ }^{f / f f}$ mice, both treated by imiquimod. $P$ values for differential expression (upregulation or downregulation) of the gene set (red) are shown. The same analysis for 2,700 mouse orthologs corresponding to the 6,900 human transcripts decreased in psoriatic epidermis (blue, the negative psoriasis signature). (B) Heatmap of expression levels of genes of the positive and negative psoriasis signature, significantly $(q<0.05)$ increased or decreased, respectively, in skin from $Z f p 36^{\triangle E P}$ mice compared with Zfp $36^{f / f}$ mice, both treated by imiquimod. Each column corresponds to a separate mouse. (C) Expression of ZFP36 in microdissected epidermis from healthy $(n=5)$ and psoriatic individuals (lesional skin) $(n=10)$ was analyzed by microarrays. Results are given as mean \pm SEM. Statistical significance $\left({ }^{*} P<0.05\right)$ was assessed by 2-tailed Mann-Whitney test compared with healthy group.

(8). We therefore added the compound SB203580 to increase the sensitivity of the mRNA decay profiling (Figure 5E). As previously demonstrated for macrophages (8, 16, 17), Tnf, Cxcl1, and Cxcl2 mRNA stability was increased in TPA-activated TTP-deficient keratinocytes. In contrast, mRNA decay of Il19 or Cxcl10 was TTP independent, while Illf6 transcripts levels were found to be stable in both groups.

Since TNF expression was dysregulated in TTP-deficient keratinocytes and given the central role of this cytokine in psoriasis, we generated mice with specific ablation of both TNF and TTP expression in keratinocytes $\left(Z f p 36^{\mathrm{A} / \mathrm{fl}} T n f^{\mathrm{A} / \mathrm{I}} \mathrm{K} 14-\mathrm{Cre}\right.$ mice, referred to here in as $Z f p 36^{\mathrm{AEP}} T n \rho^{\rho \mathrm{EP}}$ mice). These mice were then subjected to topical imiquimod treatment. The exacerbation of the skin lesions observed in $Z f p 36^{\triangle \mathrm{EP}}$ mice was abrogated in the absence of TNF production by keratinocytes (Figure 6A). This was accompanied by reduced proportions of infiltrating neutrophils, monocytes, and cytokine-producing $\gamma \delta$ T cells (Figure 6, B-D). Consistent with the role of CXCL2 as a chemoattractant for neutrophils, its mRNA expression was reduced in absence of keratinocyte-derived TNF (Figure 6E). Taken together, these results strongly support the notion that TTP-mediated $T n f$ mRNA decay in keratinocytes contributes to the control of inflammation in this psoriasis-like model. 
A

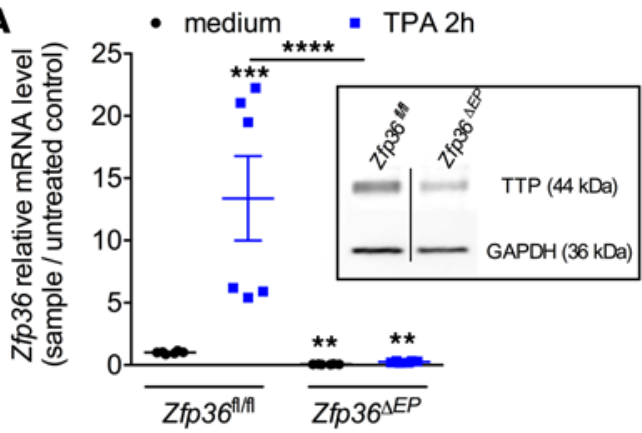

C
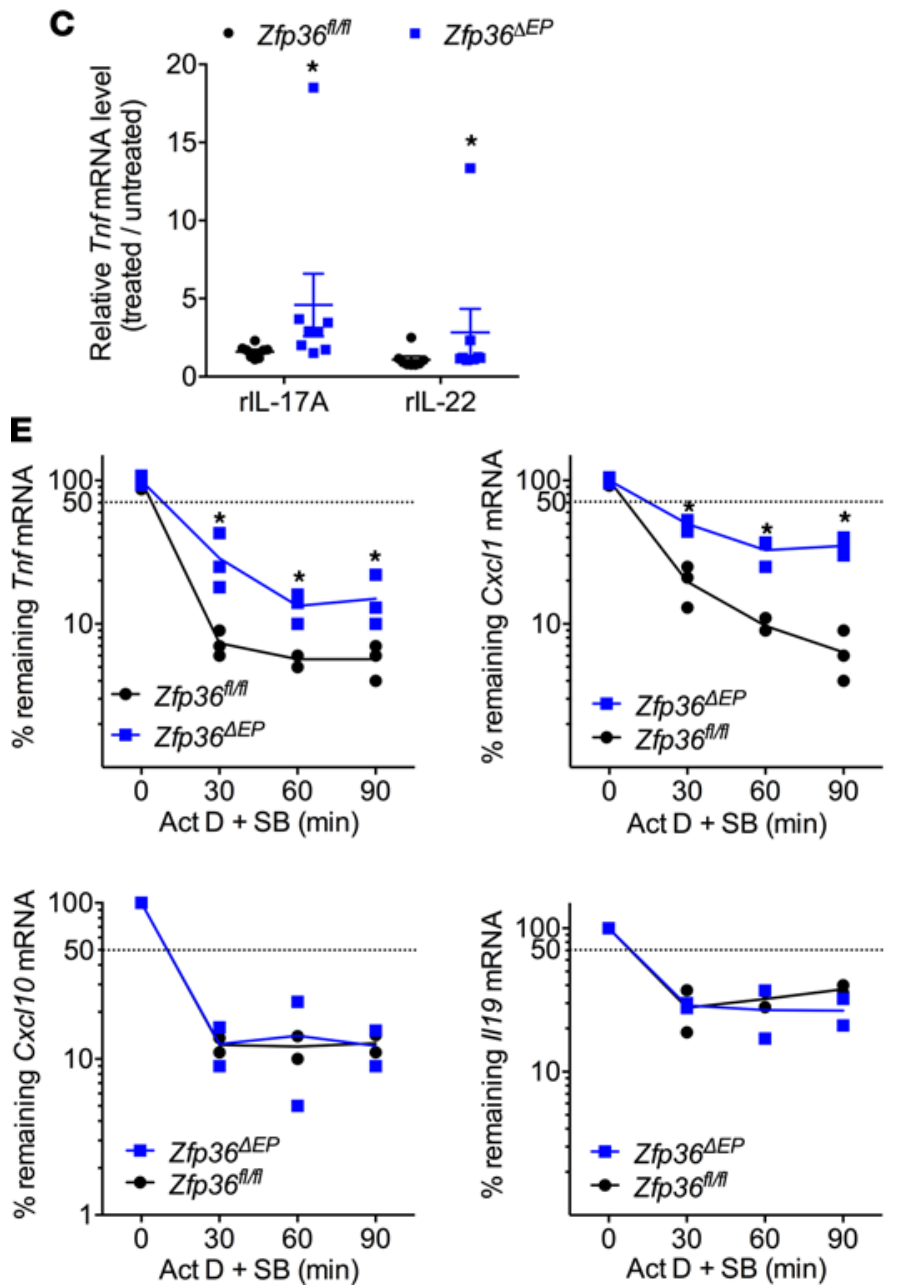
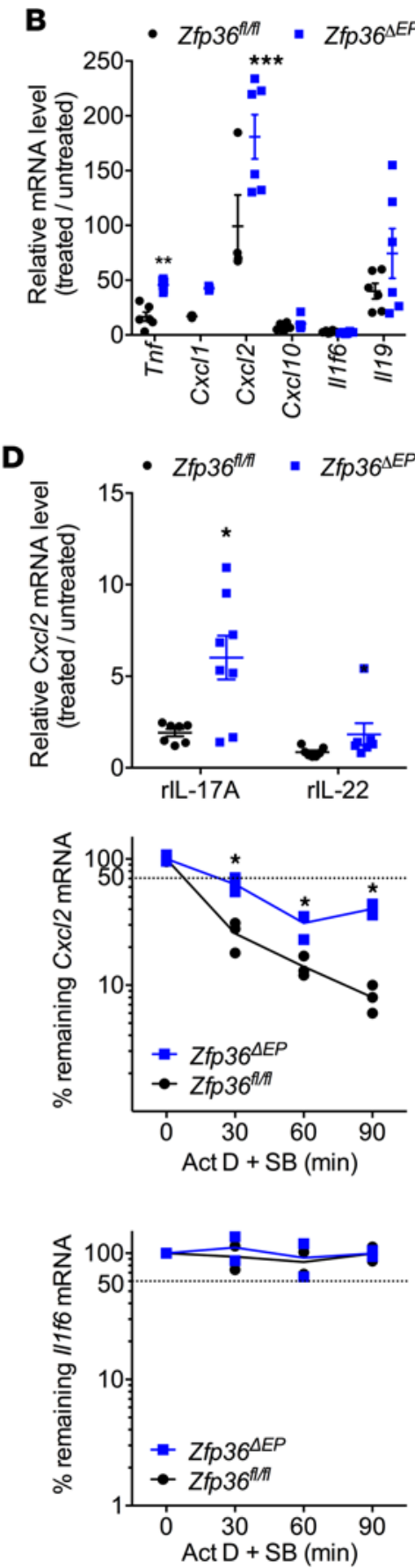

Figure 5. TTP controls the mRNA stability of many proinflammatory mediators in TPA-stimulated primary mouse keratinocytes. Primary keratinocytes isolated from $Z f p 36^{\triangle E P}$ and $Z f p 36^{f / f l}$ newborns were stimulated for 2 hours with TPA $(100 \mathrm{ng} / \mathrm{ml})$, rIL-17A (50 ng/ml), or rIL-22 (50 $\mathrm{ng} / \mathrm{ml}$ ). (A) Expression of Zfp36 was assessed by RTqPCR and Western blot (mock condition). (B) Gene expression in response to TPA stimulation. (C and D) Expression of Tnf (C) and $C x C / 2$ (D) after rIL-17A or rlL-22 stimulation. Results are given as mean \pm SEM of at least 3 independent experiments performed in triplicate. Normalized mRNA levels were expressed relative to the $Z f p$ $36^{f / f l}$ untreated condition arbitrarily set to 1. (E) mRNA halflife analysis. Two hours after TPA stimulation, actinomycin $\mathrm{D}(10 \mu \mathrm{g} / \mathrm{ml})$ and SB203580

$(1 \mu \mathrm{M})$ were added for the indicated times. SB203580 was used to abolish the inhibitory effect of p38 MAPK on TTP activation. Total RNA was extracted and analyzed by RTqPCR. Results are given as mean \pm SEM of 2 experiments performed in triplicate (a total of 6 wells from each genotype). Statistical significance $\left({ }^{*} P<\right.$ $0.05,{ }^{* *} P<0.01,{ }^{* * *} P<0.001$, ${ }^{* * * *} P<0.0001$ ) was assessed by 1-way ANOVA test with Bonferroni correction (A) or by 2-tailed Mann-Whitney test compared with the Zfp $36^{f / / f}$ group (B-E)

Spontaneous and progressive development of a skin, joint, and systemic inflammatory syndrome in Zfp36 ${ }^{\triangle E P}$ mice. As previously mentioned, whole-body deletion of $Z f p 36$ leads to the spontaneous development of a complex TNF- and IL-23-dependent inflammatory syndrome characterized by cachexia, skin lesions, arthritis, and myeloid hyperplasia $(10,11)$. Strikingly, we observed that many parameters of the "TTP deficiency syndrome" were recapitulated in $Z f p 36^{\mathrm{AEP}}$ mice as they aged. Indeed, these mice developed a spontaneous and heterogeneous pathology reminiscent of human psoriasis, exhibiting skin lesions characterized by thickening of the skin and scaly plaques over the chest, the tail, and the limbs, along with inflammation of the distal articulations, with global swelling of the paws or dactylitis (swelling of a digit), the latter being considered a hallmark of psoriatic arthritis (Figure 7A). As compared with $Z f p 36^{-1-}$ mice, $Z f p 36^{\Delta \mathrm{EP}}$ mice developed inflammatory lesions at a slower pace and with reduced severity, but eventually every $Z f p 36^{\triangle \mathrm{EP}}$ mouse exhibited skin lesions on the chest and/or the tail, along with arthritis (Figure 7, B and C). In addition, after the age of 10 
A
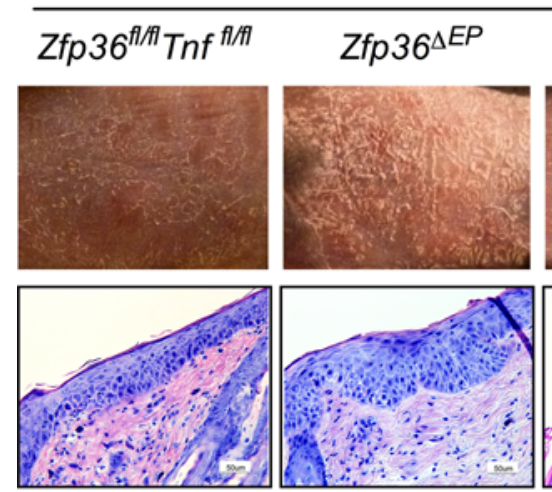

B
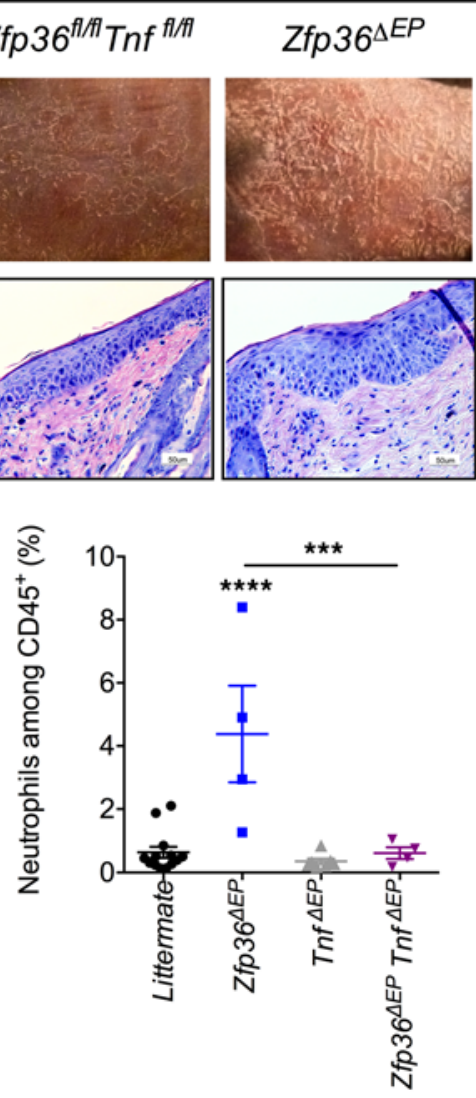

imiquimod
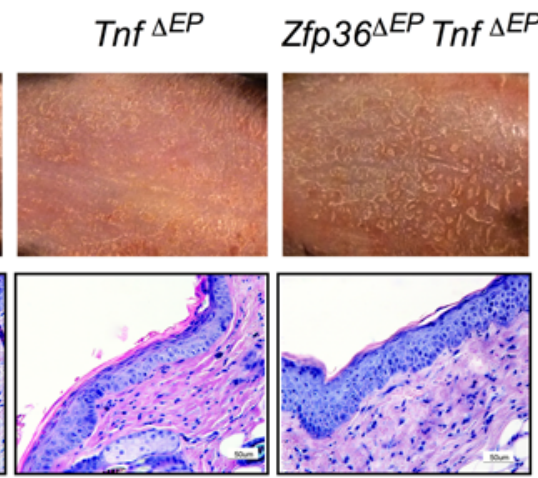

C

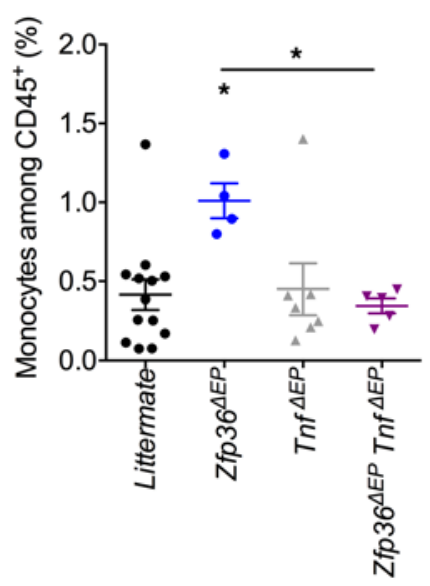

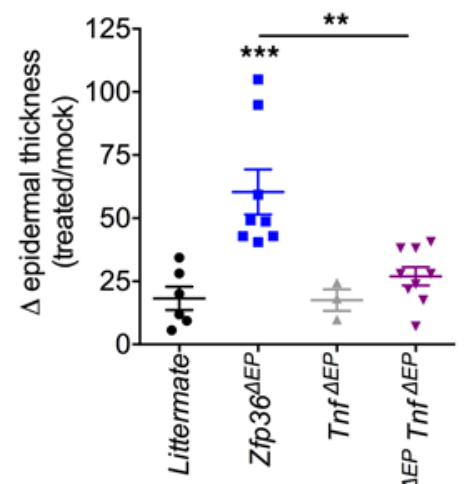

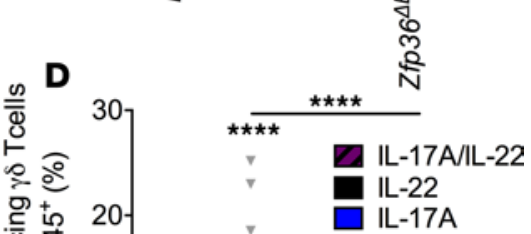

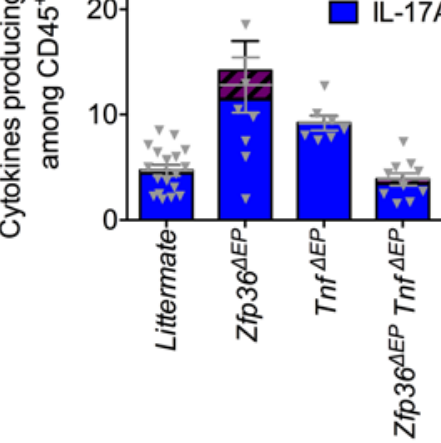
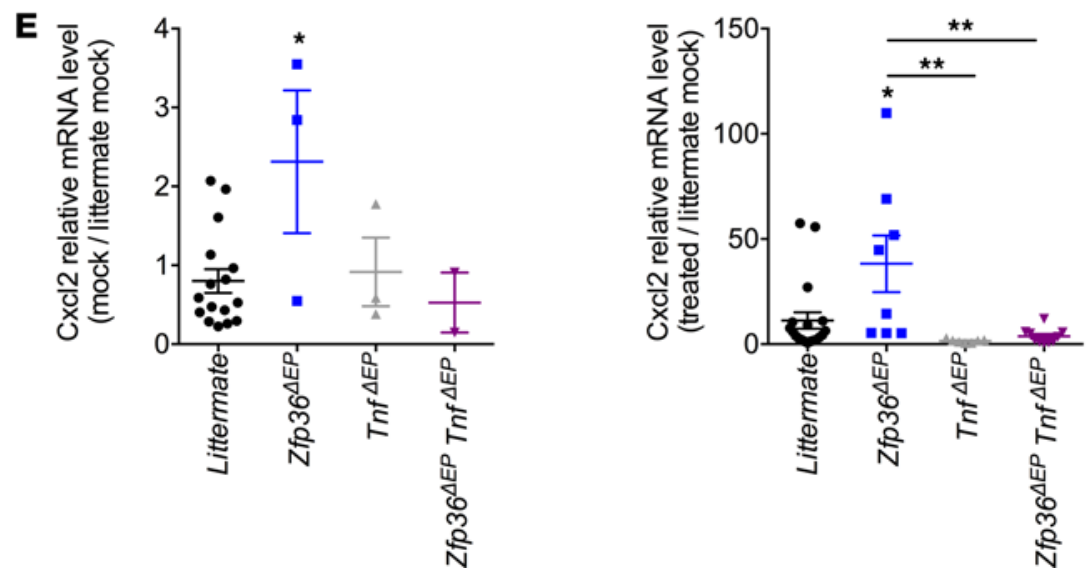

Figure 6. TNF produced by keratinocytes is critical for the control of inflammation in imiquimod-induced dermatitis. $Z f p 36^{\triangle E P}(n=8)$, $\operatorname{Tn} f^{\perp E P}(n=7)$, and

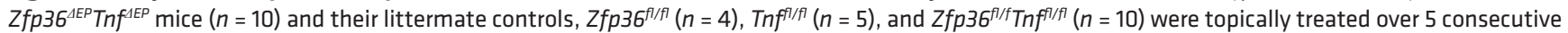
days with imiquimod. Skin samples were collected 4 hours after the last application for analysis of epidermal thickness by histology. Original magnification: $\times 200(\mathbf{A})$, cell recruitment by flow cytometry (B and C), protein production by intracellular protein staining (D), or transcript levels by RTqPCR (E). Results are given as mean \pm SEM. Statistical significance $\left({ }^{*} P<0.05\right.$, ${ }^{*} P<0.01$, ${ }^{* *} P<0.001$, ${ }^{* * *} P<0.0001$ ) was assessed by 1-way ANOVA test with Bonferroni correction compared with the littermate group (if not specified). Results are pooled from 2 independent experiments. Representative histology for mock group from each genotype is documented in Supplemental Figure 4.

weeks, $Z f p 36^{\triangle \mathrm{EP}}$ mice displayed reduced weight gain, revealing systemic inflammation (Figure 7D). Importantly, all of these clinical manifestations were found to be dependent on keratinocyte-derived TNF production, as $Z f p 36^{\triangle \mathrm{EP}} T n f^{\mathrm{EEP}}$ mice did not show any signs of spontaneous skin lesions, arthritis, or growth delay, at least up to the age of 60 weeks (Figure $7 \mathrm{E}$ ). We extended previous observations showing that $Z f p 36^{\Delta \mathrm{M}}$ mice $(8,12)$ or $Z f p 36^{\triangle \mathrm{DC}}$ mice did not display any sign of local or systemic inflammation (Supplemental Figure 4A). 
A histological study of lesional upper chest skin of $Z f p 36^{\mathrm{AEP}}$ mice displayed hallmarks of psoriasis, such as epidermal thickening (acanthosis), downward extension of the epidermal rete ridges (papillomatosis), and cellular infiltrates in the dermis (Figure 7F). Analysis of samples collected at the age of 6 to 12 months indicated that neutrophils and IL-17A-producing cells accumulated in the skin of most $Z f p 36^{\triangle \mathrm{EP}}$ mice (Figure 7, G and $\mathrm{H}$ ).

Sections of front paws indicated cellular infiltrates at the level of the enthesis of distal interphalangeal joints (see arrows, Figure 7I). To further evaluate the inflammatory lesions of the paws, we performed high-resolution CT imaging of the forelimbs. We observed that bone disruption of the distal phalanges and the first digits was present in $Z f p 36^{\triangle \mathrm{EP}}$ mice. In contrast, $Z f p 36^{\triangle \mathrm{EP}} \mathrm{Tn} f^{\mathrm{AEP}}$ mice displayed intact bone structures (Figure $7 J)$. Next, we used PET-CT scanning, using $\left[{ }^{18} \mathrm{~F}\right]$-deoxy-glucose ( $\left[{ }^{18} \mathrm{~F}\right]$-FDG). We observed high cellular uptake of $\left[{ }^{18} \mathrm{~F}\right]-\mathrm{FDG}$ around the metacarpal bones and phalanges of $Z f p 36^{\triangle \mathrm{EP}}$ mice as compared with basal uptake revealed in $Z f p 36^{f l f l}$ mice. This pattern is highly suggestive of active periarticular inflammation. In contrast, $\left[{ }^{18} \mathrm{~F}\right]$-FDG uptake was strongly decreased in paws of age-matched $Z f p 36^{\triangle \mathrm{EP}} \operatorname{Tn}{ }^{\mathrm{EEP}}$ mice (Figure $7 \mathrm{~K})$. In addition, we evaluated bone tissue remodeling processes by PET-CT using $\left[{ }^{18} \mathrm{~F}\right]-\mathrm{NaF}$. Zfp $36^{\triangle \mathrm{EP}}$ mice displayed increased uptake of the tracer at the distal interphalangeal joints and the first digit as compared with $Z f p 36^{f / f l}$ mice (Supplemental Figure 5). Taken together, these results indicate that the joint pathology developed by $Z f p 36^{\triangle \mathrm{EP}}$ mice is strongly reminiscent of human psoriatic arthritis.

Finally, we observed that G-CSF levels were increased in the sera of $Z f p 36^{\triangle \mathrm{EP}}$ and $Z f p 36^{-1-}$ mice to comparable extents (Figure 7L). Although the proportion of myeloid cells in the spleen and circulating levels of neutrophil gelatinase-associated lipocalin 2 (N-GAL), a general marker of inflammation, were also increased in $Z f p 36^{\triangle \mathrm{EP}}$ mice, they did not reach the values obtained in age-matched $Z f p 36^{-1-}$ mice (Figure 7 , $\mathrm{M}$ and $\mathrm{N}$ ). Taken together, these results indicate that absence of TTP in keratinocytes is sufficient to trigger the spontaneous development of local and systemic aspects of the "TTP deficiency syndrome."

\section{Discussion}

Inflammation is a fundamental adaptation of the organism to the loss of tissue homeostasis with many important physiological roles, including host defense and tissue repair. Classically, immune sentinel cells, such as Langerhans cells, macrophages, or DCs, have been regarded as the primary sources of cytokines and chemokines in response to microbial or danger stimuli. However, at epithelial surfaces or within organs, initiation, amplification, and resolution of inflammation involves complex interactions with other immune populations (such as innate and memory lymphoid cells) and nonhematopoietic cells (such as stromal, epithelial, and endothelial cells). Our results uncover a central role of TTP expression by keratinocytes rather than immune cells in the control of skin inflammation in the imiquimod-induced psoriasis-like model. In addition, most features of the "TTP deficiency syndrome" were recapitulated in $Z f p 36^{1 E P}$ mice. Indeed, these mice developed a spontaneous pathology with hyperproliferative dermatitis, arthritis of distal joints, and systemic inflammation. In contrast, neither myeloid- $(8,12)$ nor DC-restricted $Z f p 36$ ablation was sufficient to drive spontaneous inflammation (Supplemental Figure 5A). As clinical manifestations occurred more rapidly and were more severe upon constitutive TTP ablation, expression within multiple cellular compartments is likely to contribute to the control of systemic inflammation (18). However, TTP has multiple targets, including IL-10 $(19,20)$. Hence, in specific contexts, TTP might actually decrease the antiinflammatory properties of regulatory subpopulations such as Tregs or myeloid-derived suppressor cells. In keratinocytes, we show that TTP controls the mRNA stability of classical targets, such as Tnf, Cxcl1, and Cxcl2. We provide genetic evidence that TNF produced by keratinocytes plays a dominant role in local and systemic inflammation. This cytokine has multiple effects in the skin. For example, it may act in an autocrine/paracrine fashion to trigger epithelial cell death (21) and IL-24 production (22). Treatment of patients suffering from psoriatic lesions with etanercept, a soluble TNF receptor that blocks TNF signaling, decreases DC activation and IL-23p19 production in these lesions, suggesting that it acts upstream of other mediators (23). Importantly, mice harboring a deletion in the $3^{\prime}$ AREs of the TNF mRNA (Tnf $f^{A R E}$ mice) develop ileitis and chronic polyarthritis but no apparent skin pathology $(24,25)$. Although we found TNF to be critical, these data suggest that other TTP targets in keratinocytes might be important to drive the spontaneous pathology developed by $Z f p 36^{1 E P}$ mice.

Most animal models of "spontaneous" psoriasis are based on transgenic overexpression of pathogenic cytokines (e.g., K5.TGF- $\beta$, K14.IL-12B, K14.IL-17A) or constitutively active signaling pathways (e.g., K5.STAT3C, K14.AREG) (K5 and K14 referring to the promotor controlling the transgenic mouse line, Keratin5 and Keratin14, respectively). We therefore propose that $Z f p 36^{\backslash E P}$ mice might represent a valuable model to explore the role of environmental triggers in psoriasis development. Furthermore, arthritis is rare- 

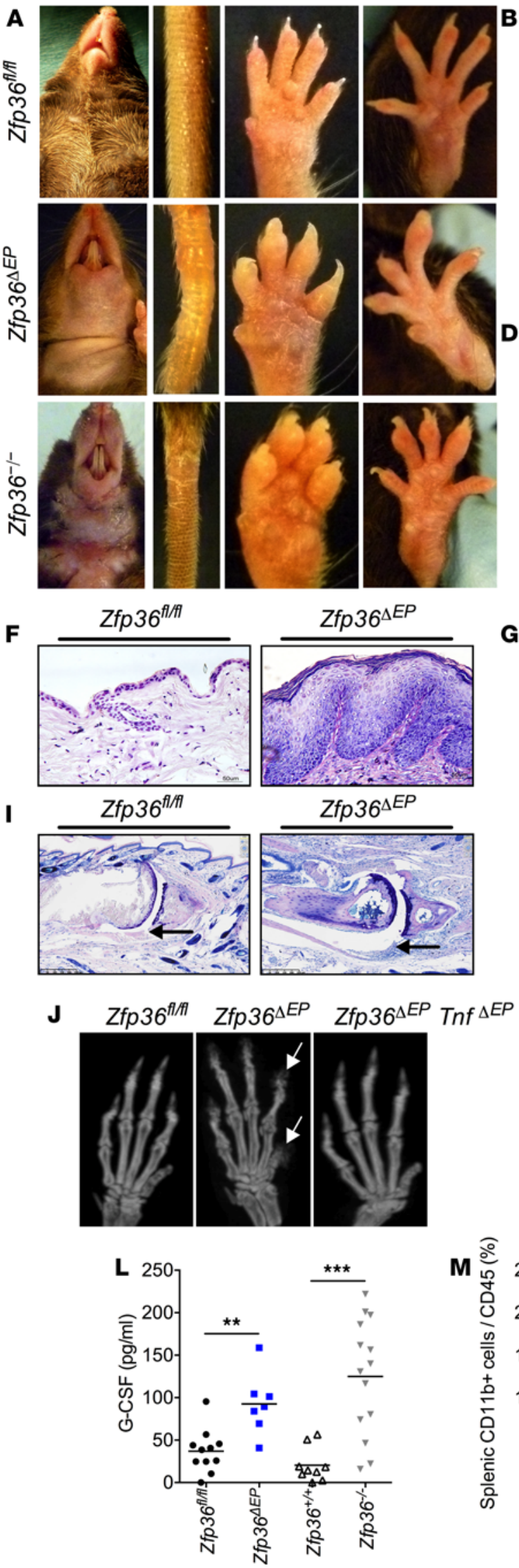
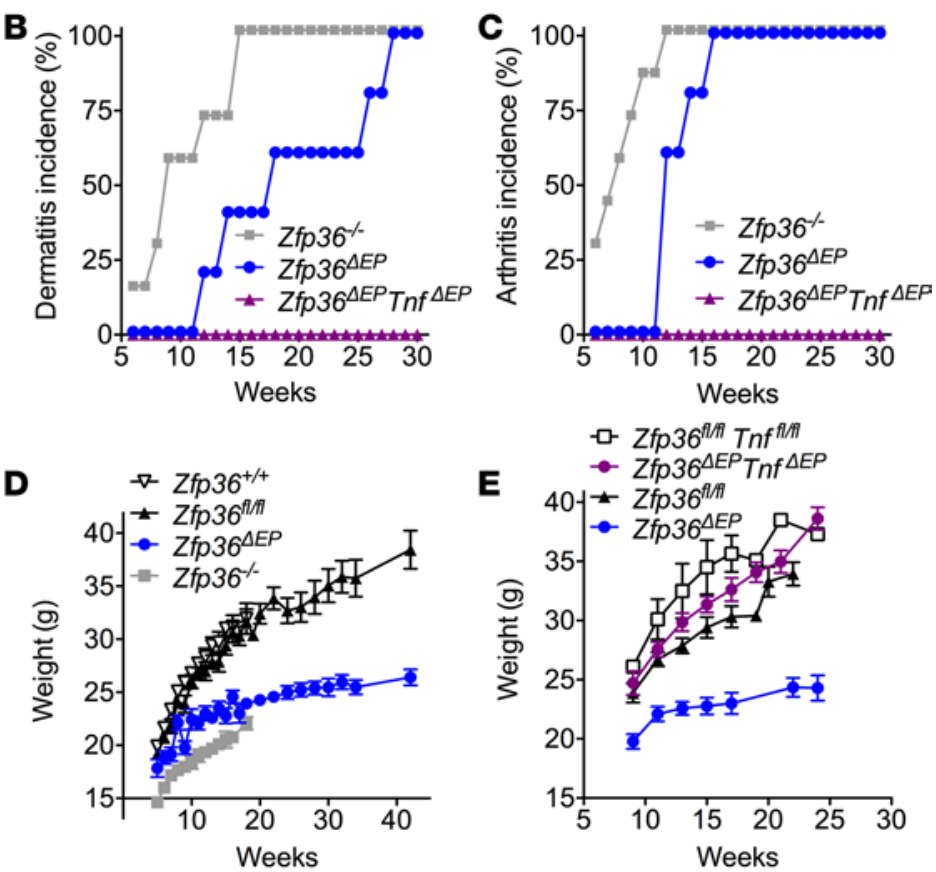

G
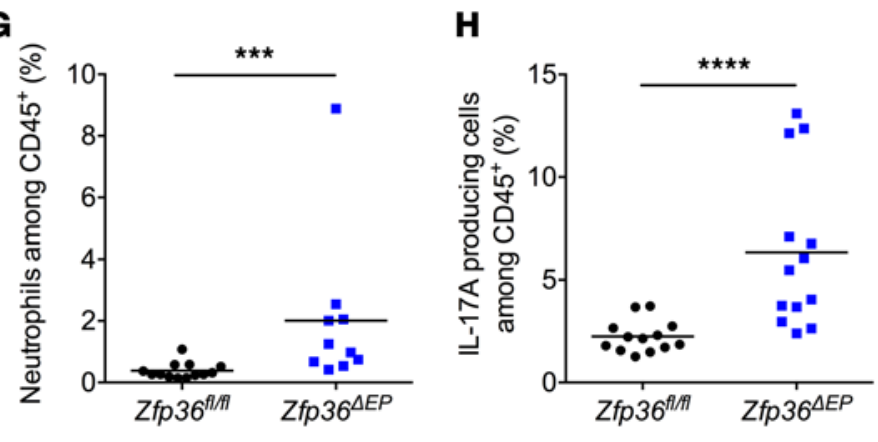
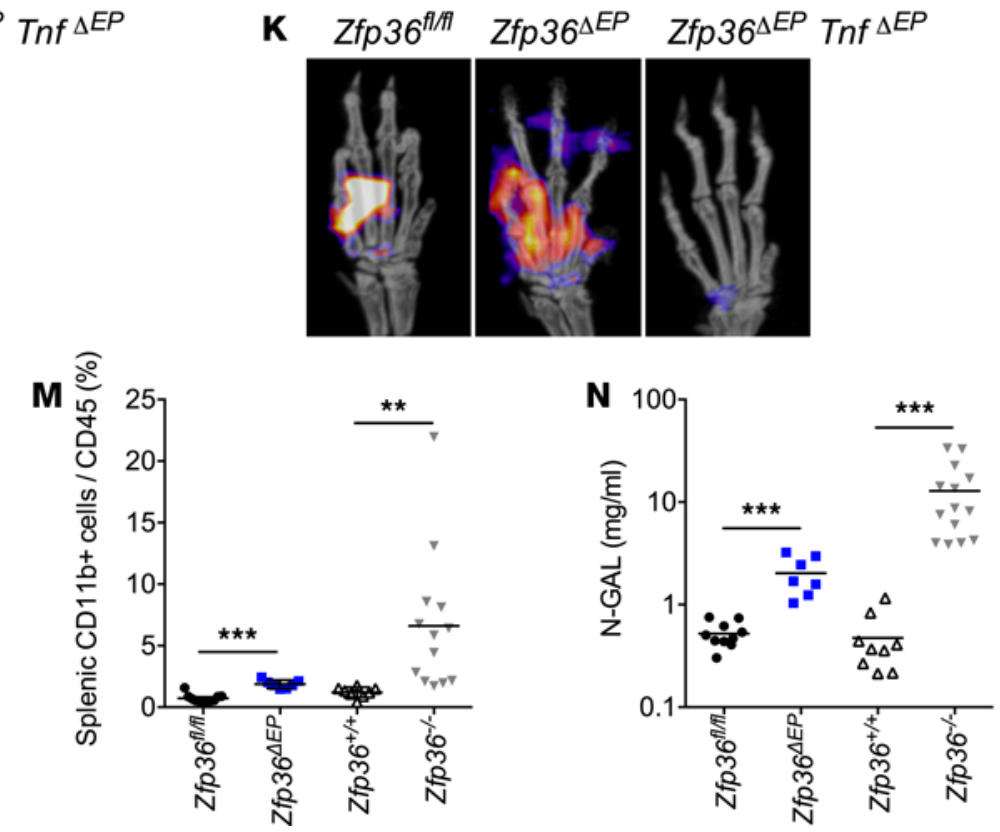
Figure 7. Loss of tristetraprolin expression by keratinocytes leads to the spontaneous development of local and systemic inflammation. Zfp $36^{\triangle E P}$ ( $n=17), Z f p 36^{-/-}(n=7)$, and $Z f p 36^{\perp E P} T n f^{A E P}$ mice $(n=7)$ and their respective littermate controls, $Z f p 36^{f f l}(n=17), Z f p 36^{+/+}(n=7)$, and $Z f p 36^{f / / f I} T n f^{f l / f l}$ mice $(n=4)$, were monitored weekly for clinical features. (A) Phenotypical presentation: macroscopic views of representative Zfp36 $6^{4 E P}, Z f p 36^{f / f l}$, and Zfp36-/- mice, showing, from left to right, neck skin, tail, front paw, and back paw. (B) Incidence of skin lesions. (C) Incidence of arthritis. (D and E) Weight curves. (F-H) Features of skin inflammation of 10- to 12-month-old male mice. (F) Histological view of MGG-stained section of upper thoracic skin samples (representative of 17 animals by group). (G) Proportion of neutrophils and (H) IL-17A-producing cells in the skin of $Z \mathrm{fp} 36^{4 \mathrm{EP}}(n=13)$ and

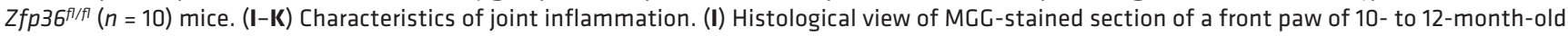
male mice. Arrows indicate the entheseal regions (representative of 17 animals by group). (J) High-resolution CT scan and (K) PET-CT scan of the front paw of 6-month-old male mice injected with $\left[{ }^{18} \mathrm{~F}\right]-\mathrm{FDG}$ (1 representative of 4 mice). Arrows indicate bone disruption. (L) G-CSF levels in the serum, (M) proportion of myeloid cells (CD45+CD19-CD3-CD11 b) in the spleen, and (N) N-CAL levels in the sera (note the log scale for $y$ axis) of Zfp36 $6^{4 E P}(n=7)$ and

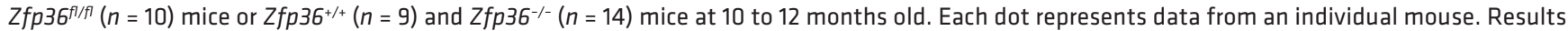
are given as mean \pm SEM. Statistical significance $\left({ }^{*} P<0.05,{ }^{* *} P<0.01,{ }^{* *} P<0.001\right)$ was assessed by 2-tailed Mann-Whitney compared with the Zfp36 $6^{f / f l}$ mock group (if not specified) ${ }^{* *} P<0.01$, ${ }^{* * *} P<0.001$, ${ }^{* * *} P<0.0001$ ). Results are representative of 2 experiments (see also Supplemental Figure 5). Original magnification: $\times 200(\mathbf{F}$ and $\mathbf{I})$.

ly observed in mouse psoriasis models. One notable exception is the inducible epidermal deletion of JunB and its functional companion c-Jun in adult mice that leads to a rapid and severe phenotype resembling the histological and molecular hallmarks of psoriasis, including arthritic lesions (26). More recently, Yamamoto et al. showed that autoimmune arthritis-prone F759 mice harboring the K5-Stat3C transgene not only had aggravated skin lesions, but also spontaneously developed arthritis with high penetrance (27). We show that dysregulated keratinocyte-derived TNF is responsible for this pathological link. Together with these previous reports, the present results therefore indicate that joint involvement in psoriatic patients could be secondary to epidermal inflammation. As TNF is not readily detected in the sera of $Z f p 36^{1 E P}$ mice, it is still unclear which circulating factors could promote systemic and distant pathological features. For example, other factors, such as lipocalin 2, an iron-binding protein with antimicrobial properties, could participate in this process (28). Indeed, we observed elevated concentrations of this mediator in the sera of $Z f p 36^{4 E P}$ mice (Figure $7 \mathrm{~N}$ ).

We observed reduced expression of ZFP36 mRNA in epidermal cells from psoriatic patients. This suggests that TTP expression might be compromised in keratinocytes of psoriatic patients, thereby contributing to exacerbated inflammation. Previous transcriptomic data confirmed reduced expression of ZFP36 mRNA in involved psoriasis skin as compared with healthy individuals (29). Interestingly, in this data set, expression was also significantly lower in uninvolved skin as compared with healthy samples, suggesting that it could be a primary event contributing to the onset of the pathology. However, this was not observed in another transcriptome study (30). Several factors could contribute to these discrepancies. ZFP36 expression is highly regulated in the context of inflammation and cell differentiation, and psoriasis is a dynamic disease, as chronic plaques are the seat of inflammatory bursts (31). Furthermore, pharmacological treatment used in the context of psoriasis, such as glucocorticoids, is known to promote ZFP36 expression (32).

Although several polymorphisms are found in the general populations (33), genome-wide association studies of psoriatic patients did not identify susceptibility variants in the ZFP36 regions. Hence, decreased ZFP36 expression in the skin of psoriatic patients could be secondary to perturbation of other pathways. For example, decreased AP-1 activity (26) could alter ZFP36 expression in keratinocytes, as these pathways are intimately linked (34). Our preliminary data also indicate that $Z f p 36$ expression is reduced in the skin of K5-STAT3C transgenic mice, suggesting that chronic activation of this pathway might repress Zfp36 gene expression in keratinocytes. Future work will aim at understanding the regulation and the physiological role of TTP in these cells. As p38 MAPK-dependent phosphorylation of TTP at Ser ${ }^{52}$ and Ser ${ }^{178}$ leads to inactivation but also protection from proteasomal degradation, leading to protein accumulation both in vitro and in vivo $(35,36)$, it would be important to explore not only the protein expression, but also the functional status of TTP in physiological and pathological settings. Overexpression of endogenous TTP by knocking out an instability motif in its mRNA (TTP ${ }^{A \mathrm{ARE}}$ mice) was recently shown to be highly efficient in protecting mice from imiquimod-induced skin inflammation and collagen antibody-induced arthritis (37). In addition, mice harboring a genetically modified unphosphorylable form of TTP are in turn protected against collagen-induced arthritis (38). Finally, inhibition of insulin receptor substrate-1 by topical application of antisense oligonucleotides in a randomized pilot trial in psoriasis was suggested to mediate its therapeutic effect by preventing the binding of TTP to 14-3-3 protein, thereby increasing its activity (39). Collectively, our results indicate that TTP plays a major role in skin immune homeostasis. Hence, strategies that would increase TTP expression or its activity in keratinocytes could therefore have important therapeutic potential in the context of human psoriasis. 


\section{Methods}

For additional details, see the Supplemental Methods.

Mice. Zfp36-deficient mice (Zfp36 $\left.6^{-/}\right)$and LoxP-flanked Zfp36 mice (Zfp36 $\left.6^{l / f}\right)$ mice on a C57BL/6 background were previously described $(10,12)$. LysM-Cre, CD11c-Cre, and K14-Cre mice on a C57BL/ 6 background were purchased from The Jackson Laboratory. LoxP-flanked $\operatorname{Tnf}$ mice ( $\operatorname{Tn} f^{(1 / f)}$ ) on a C57BL/6 background were previously described (40). Cell-specific $Z f p 36^{-/-}$mice were generated by crossing the $Z f p 36^{f / f l}$ mice (female) with mice (male) expressing Cre recombinase under the control of the murine M lysozyme promoter $\left(Z f p 36^{1 M}\right.$ ), the murine integrin $\alpha \mathrm{X}$ promoter $\left(Z f p 36^{1 D C}\right)$, or the human keratin 14 promoter $\left(Z f p 36^{4 E P}\right)$. The double-conditional $Z f p 36^{\lfloor E P} T n f^{A E P}$ mice were obtained by mating $Z f p 36^{\triangle E P}$ mice with $\operatorname{Tnf}^{l / f l}$ mice. All experiments were performed using littermates as controls. All mice were bred and maintained in a specific pathogen-free animal facility.

Imiquimod-induced dermatitis. Six-week-old female mice were anesthetized by i.p. injection of ketamine and xylazine, shaved on the abdomen with an electrical shaver, and chemically depilated with Veet hair remover. Mice were topically treated with a cream containing 5\% imiquimod (Aldara, 3M pharmaceuticals) over 5 consecutive days ( $6.25 \mathrm{mg}$ imiquimod per day). Mice were sacrificed, and skin samples were prepared for histology, gene expression, or flow cytometry analysis.

Flow cytometry analysis. Skin samples were incubated for 16 hours with dispase II (Sigma-Aldrich, $1 \mathrm{mg} / \mathrm{ml}$ ) and then with collagenase IV (Worthington, $1 \mathrm{mg} / \mathrm{ml}$ ) and DNase I (Sigma-Aldrich, $100 \mu \mathrm{g} / \mathrm{ml}$ ) for $45 \mathrm{~min}$ utes at $37^{\circ} \mathrm{C}$. Cells were separated on Percoll $20 \%$ and stained for CD45-FITC; IL-22-PE; Ly6G-PerCP-Cy5.5; $\gamma \delta$ TCR-PerCP-eFluor710; CD127-PE-Cy7; IL-17A-APC; CD3-, CD19-, B220-, and Gr1-APC-Cy7; CD11b-, CD90.2-AF700, Ly6C-, or CD3-BV421 (see Supplemental Table 1 for manufacturers and references). Cells were incubated for 2 hours with PMA $(25 \mathrm{ng} / \mathrm{ml})$, ionomycin $(500 \mathrm{ng} / \mathrm{ml})$, and brefeldin A $(10 \mu \mathrm{g} / \mathrm{ml})$ and processed for intracellular staining using the Intracellular Fixation \& Permeabilization kit (eBiosciences). Data were collected on a BD LSRII Fortessa and analyzed with BD FACSDiva software.

Gene expression. Total RNA was extracted with the RNeasy Minikit (Qiagen) and reverse-transcribed with the High-Capacity cDNA Archive Kit (Applied Biosystems). cDNA was amplified using SYBR green or TaqMan probes. Data were processed out using DataAssist software (Thermo Fisher Scientific) with the RQ analysis module. Relative mRNA levels $\left(2^{-\Delta \Delta \mathrm{Ct}}\right)$ were determined by comparing (a) the cycle thresholds (Ct) for the gene of interest and 3 calibrator genes $(\Delta \mathrm{Ct}), A c t b, G a p d h$, and Hprt, and (b) $2^{-\Delta C t}$ values for imiquimod-treated group compared with mock-treated group. Ct values were limited to 35 cycles. Primer sequences are available in Supplemental Table 2.

Histology. Mouse tissues were fixed in 4\% paraformaldehyde. Skin samples were directly paraffin embedded. Joints were first decalcified. Sections $(6 \mu \mathrm{m})$ were stained with May Grünwald Giemsa or for Ki67. Epidermal thickness was measured at 200-fold magnification; a mean of 3 measures was calculated for each sample. Ki67 ${ }^{+}$cells were quantified using VisioPharm Software.

Western blot. Mouse skin samples were lysed in RIPA buffer (Igepal CA-630 0.08 mM [Sigma-Aldrich], sodium deoxycholate $0.5 \%$ [Sigma-Aldrich], sodium dodecyl sulfate $0.1 \%$ [Sigma-Aldrich], Complete EDTAfree protease inhibitor cocktail [Roche Applied Science], and PhosSTOP [Roche Applied Science]). Protein extracts were cleared by centrifugation and then reduced with laemmli buffer (Tris- $\mathrm{HCl}$ [pH 6.8] $300 \mathrm{mM}$; sodium dodecyl sulfate 10\%; glycerol 50\%; bromophenol blue $0.005 \%$ ) containing 2-mercaptoethanol 5\% (Sigma-Aldrich) for 10 minutes at $95^{\circ} \mathrm{C}$ and immediately snap frozen. The following primary antibodies were used: mouse anti-GAPDH (clone 6C5; Meridian Life Science). The rabbit anti-TTP (Carp-3) was provided by William Rigby (Dartmouth-Hitchcock Medical Center, Lebanon, New Hampshire, USA) (41).

Cell culture. Mouse primary keratinocytes were isolated from newborn mice (24-72 hours after birth) as described by Li et al. (42). Cells were maintained in complete Keratinocyte Growth Medium II (Promocell $\mathrm{GmbH})$ at $36^{\circ} \mathrm{C}$ and $7 \% \mathrm{CO}_{2}$.

Microarrays. Total RNA from skin samples was extracted with the RNeasy Mini Kit (Qiagen). 200 ng total RNA were then amplified with the GeneChip WT PLUS Reagent Kit and labeled with the GeneChip WT Terminal Labeling Kit (Affymetrix). The resulting complementary RNAs were hybridized on the GeneChip Mouse Gene 2.0 ST Array (Affymetrix) according to the manufacturer's protocol. Microarray data were normalized by the Robust Multi-Average method (43) by using affy $\mathrm{R}$ package (44). Differentially expressed genes were identified by using limma $\mathrm{R}$ package (45), which uses an empirical Bayesian approach to estimate variances in moderated $t$ tests. Raw $P$ values were adjusted for multiple testing using the Storey $q$ value method to control the FDR (46). Gene Expression Omnibus repository accession number for these data is GSE90450. 
Gene expression on human epidermis. Skin biopsies (5-mm diameter) were obtained from 10 patients with psoriasis and 5 age- and sex-matched healthy volunteers. The epidermis was isolated by laser-capture microdissection using the Arcturus XT (MDS Analytical Technologies) system (47) followed by RNA isolation with the Arcturus Picopure RNA Isolation Kit. 10 ng RNA were amplified with the ExpressArt Kit and labeled with One-color microarray-based Gene Expression Analysis Low Input Quick Amp WT Labeling (Agilent Technologies) and hybridized with the Human Gene Expression Microarray 8x60K chip (Agilent Technologies). Two sets of samples ( 3 lesional psoriatic and 3 healthy epidermis samples in set 2) were processed and normalized separately. Microarray data were background corrected with the normal-exponential convolution model (48) and normalized with the quantile approach (49) using the limma R package (45). Gene Expression Omnibus repository accession numbers for these data are GSE68923 and GSE68937 for human epidermis.

Cytokines production. The concentration of G-CSF and N-GAL were determined in sera by ELISA (R\&D Systems).

PET-CT imaging of ${ }^{18} \mathrm{~F}$-FDG. Joint inflammation was assessed by $\left[{ }^{18} \mathrm{~F}\right]-\mathrm{FDG}$ PET imaging. $\left[{ }^{18} \mathrm{~F}\right]$-FDG was synthesized at the PET/Biomedical Cyclotron Unit of the Nuclear Medicine Department at ULB-Hôpital Erasme. Mice were fasted overnight. They were injected intravenously (in the lateral tail vein) with 3.9-5.5 MBq $\left[{ }^{18} \mathrm{~F}\right]-\mathrm{FDG}$ and kept under isoflurane anesthesia for 10 minutes to limit tracer uptake within skeletal muscle and brown adipose tissue (3). $\left[{ }^{18} \mathrm{~F}\right]$-FDG PET-CT imaging was performed on a preclinical PET-CT scanner (nanoScan PET/CT with Nucline v2.01 [019.0000]). CT images were obtained for localization as well as for attenuation and scatter correction of PET images (CT acquisition parameters: maximal field of view, $50 \mathrm{kV}, 520 \mu \mathrm{A}, 300 \mathrm{~ms}$ per projection, 480 projections per rotation, 4-to-1 frame binning, providing an isotropic reconstructed voxel size of $251 \mu \mathrm{m}$ ). PET images were acquired for 15 minutes, 60 minutes after injection (3-to-1 coincidence mode, normal count rate), and reconstructed using a 3-dimensional Ordered Subsets Expectation Maximization algorithm (TeraTomo, Mediso Ltd., with 4 iterations, 6 subsets, intermediate regularization setting, median filtering period defined from iteration counts and spike filter) with a voxel size of $0.4 \mathrm{~mm}$. All PET images were also corrected for random counts, dead time, and decay. Finally, high-resolution CT acquisitions with an isotropic reconstructed voxel size of $40 \mu \mathrm{m}$ were performed onto the anterior legs of the mice (acquisition parameters were maximum zoom, $50 \mathrm{kV}, 520 \mu \mathrm{A}, 300 \mathrm{~ms}$ per projection, 480 projections per rotation, 4-to-1 frame binning; reconstruction with a Ramlak filter and 8 regular samples). The mice were maintained under anesthesia throughout the imaging procedure.

Statistics. Results are expressed as mean \pm SEM. Statistical significance was assessed using the 2-tailed Mann-Whitney test or the 1-way ANOVA test with Bonferroni correction (GraphPad Prism 6.0). Results were considered significant at $P<0.05$.

Study approval. All animal studies were approved by the institutional animal care and local committee for animal welfare of the Biopole ULB Charleroi. Skin biopsies from patients with psoriasis and age- and sex-matched healthy volunteers were obtained after informed consent, which was included as part of the FRADAPSO study, and were approved by the Committee for Protection of Persons Nord-Ouest IV, under number 2010-A00856-33.

\section{Author contributions}

MA, AA, and LVM conducted most of the experiments. CL, DM, DSS, SF, GD, and GVS contributed to some experiments. SN, PJB, and DD provided input for research design and interpretation. MA, AA, SG, and LVM designed the study and wrote the manuscript. SG obtained the funding and supervised the work. All authors were involved in critically revising the manuscript for important intellectual content. All authors had full access to the data and approved the manuscript before it was submitted by the corresponding author.

\section{Acknowledgments}

This work was supported by the Fonds National de la Recherche Scientifique (FRS-FNRS, Belgium)/ Télévie, an Interuniversity Attraction Poles Programme of the Belgian Federal Science Policy and by the European Regional Development Fund (ERDF) and the Walloon Region (Wallonia-Biomed portfolio, 411132-957270). MA is supported by the Fondation Rose et Jean Hoguet. SG is a senior research associate and CL is a research fellow at the FRS-FNRS. PJB is supported by the Intramural Research Program of the National Institute of Environmental Health Sciences, NIH. DD, DSS, DM, and SF are supported by funding from the European Genomic Institute for Diabetes (ANR-10-LABX-46), from the European 
Commission, from the Fondation pour la Recherche Médicale (Human Physiopathology), and from the Fondation de France. We thank M. Nguyen and S. Thomas for technical assistance. The authors are grateful to Digital Image Analysis in Pathology - CMMI for technical assistance. The CMMI is supported by the Fondation ULB, the Fonds Erasme, "Association Vinçotte Nuclear" (AVN), the ERDF, and the Walloon Region. The authors thank N. Passon (CMMI, ULB) for technical work and the Cyclotron team (Hôpital Erasme) for radioisotope production.

Address correspondence to: Stanislas Goriely, Institute for Medical Immunology, 8 rue Adrienne Bolland, B-6041 Charleroi-Gosselies, Belgium. Phone: 32.2.650.9586; E-mail: stgoriel@ulb.ac.be.

1. Perera GK, Di Meglio P, Nestle FO. Psoriasis. Annu Rev Pathol. 2012;7:385-422.

2. Boehncke WH, Schön MP. Psoriasis. Lancet. 2015;386(9997):983-994.

3. Mease PJ. Inhibition of interleukin-17, interleukin-23 and the TH17 cell pathway in the treatment of psoriatic arthritis and psoriasis. Curr Opin Rheumatol. 2015;27(2):127-133.

4. Lande R, et al. Plasmacytoid dendritic cells sense self-DNA coupled with antimicrobial peptide. Nature. 2007;449(7162):564-569.

5. Maeda S, Hayami Y, Naniwa T, Ueda R. The Th17/IL-23 axis and natural immunity in psoriatic arthritis. Int J Rheumatol. 2012;2012:539683.

6. Sherlock JP, et al. IL-23 induces spondyloarthropathy by acting on ROR- $\mathrm{t}^{+} \mathrm{CD} 3^{+} \mathrm{CD} 4^{-} \mathrm{CD} 8^{-}$entheseal resident $\mathrm{T}$ cells. Nat Med. 2012;18(7):1069-1076.

7. Benham $\mathrm{H}$ et al. Interleukin-23 mediates the intestinal response to microbial $\beta$-1,3-glucan and the development of spondyloarthritis pathology in SKG mice. Arthritis Rheumatol. 2014;66(7):1755-1767.

8. Kratochvill F, et al. Tristetraprolin-driven regulatory circuit controls quality and timing of mRNA decay in inflammation. $M o l$ Syst Biol. 2011;7:560.

9. Rabani M, et al. High-resolution sequencing and modeling identifies distinct dynamic RNA regulatory strategies. Cell. 2014;159(7):1698-1710.

10. Taylor GA, et al. A pathogenetic role for TNF alpha in the syndrome of cachexia, arthritis, and autoimmunity resulting from tristetraprolin (TTP) deficiency. Immunity. 1996;4(5):445-454.

11. Molle C, et al. Tristetraprolin regulation of interleukin 23 mRNA stability prevents a spontaneous inflammatory disease. $J$ Exp Med. 2013;210(9):1675-1684.

12. Qiu LQ, Stumpo DJ, Blackshear PJ. Myeloid-specific tristetraprolin deficiency in mice results in extreme lipopolysaccharide sensitivity in an otherwise minimal phenotype. J Immunol. 2012;188(10):5150-5159.

13. Cai Y, et al. Pivotal role of dermal IL-17-producing $\gamma \delta$ T cells in skin inflammation. Immunity. 2011;35(4):596-610.

14. Prenzler F, Fragasso A, Schmitt A, Munz B. Functional analysis of ZFP36 proteins in keratinocytes. Eur J Cell Biol. 2016;95(8):277-284

15. Marichal T, et al. Guanine nucleotide exchange factor RABGEF1 regulates keratinocyte-intrinsic signaling to maintain skin homeostasis. J Clin Invest. 2016;126(12):4497-4515.

16. Carballo E, Lai WS, Blackshear PJ. Feedback inhibition of macrophage tumor necrosis factor-alpha production by tristetraprolin. Science. 1998;281(5379):1001-1005.

17. Datta S, et al. Tristetraprolin regulates CXCL1 (KC) mRNA stability. J Immunol. 2008;180(4):2545-2552.

18. Carballo E, Gilkeson GS, Blackshear PJ. Bone marrow transplantation reproduces the tristetraprolin-deficiency syndrome in recombination activating gene-2 (-/-) mice. Evidence that monocyte/macrophage progenitors may be responsible for TNFo overproduction. J Clin Invest. 1997;100(5):986-995.

19. Stoecklin G, et al. Genome-wide analysis identifies interleukin-10 mRNA as target of tristetraprolin. J Biol Chem. 2008;283(17):11689-11699.

20. Tudor C, et al. The p38 MAPK pathway inhibits tristetraprolin-directed decay of interleukin-10 and pro-inflammatory mediator mRNAs in murine macrophages. FEBS Lett. 2009;583(12):1933-1938.

21. Bonnet MC, et al. The adaptor protein FADD protects epidermal keratinocytes from necroptosis in vivo and prevents skin inflammation. Immunity. 2011;35(4):572-582.

22. Kumari S, et al. Tumor necrosis factor receptor signaling in keratinocytes triggers interleukin-24-dependent psoriasis-like skin inflammation in mice. Immunity. 2013;39(5):899-911.

23. Gottlieb $\mathrm{AB}$, et al. TNF inhibition rapidly down-regulates multiple proinflammatory pathways in psoriasis plaques. $J$ Immunol. 2005;175(4):2721-2729.

24. Kontoyiannis D, Pasparakis M, Pizarro TT, Cominelli F, Kollias G. Impaired on/off regulation of TNF biosynthesis in mice lacking TNF AU-rich elements: implications for joint and gut-associated immunopathologies. Immunity. 1999;10(3):387-398.

25. Kontoyiannis D, et al. Genetic dissection of the cellular pathways and signaling mechanisms in modeled tumor necrosis factor-induced Crohn's-like inflammatory bowel disease. J Exp Med. 2002;196(12):1563-1574.

26. Zenz R, et al. Psoriasis-like skin disease and arthritis caused by inducible epidermal deletion of Jun proteins. Nature. 2005;437(7057):369-375.

27. Yamamoto $\mathrm{M}$, et al. Psoriatic inflammation facilitates the onset of arthritis in a mouse model. J Invest Dermatol. 2015;135(2):445-453.

28. Uluçkan Ö, Wagner EF. Chronic systemic inflammation originating from epithelial tissues. FEBS J. 2017;284(4):505-516.

29. Yao Y, et al. Type I interferon: potential therapeutic target for psoriasis? PLoS One. 2008;3(7):e2737.

30. Li B, et al. Transcriptome analysis of psoriasis in a large case-control sample: RNA-seq provides insights into disease mechanisms. J Invest Dermatol. 2014;134(7):1828-1838. 
31. Christophers E, Metzler G, Röcken M. Bimodal immune activation in psoriasis. Br J Dermatol. 2014;170(1):59-65.

32. Ishmael FT, et al. Role of the RNA-binding protein tristetraprolin in glucocorticoid-mediated gene regulation. J Immunol. 2008;180(12):8342-8353.

33. Carrick DM, et al. Genetic variations in ZFP36 and their possible relationship to autoimmune diseases. J Autoimmun. 2006;26(3):182-196.

34. Amit I, et al. A module of negative feedback regulators defines growth factor signaling. Nat Genet. 2007;39(4):503-512.

35. Brook M, et al. Posttranslational regulation of tristetraprolin subcellular localization and protein stability by p38 mitogen-activated protein kinase and extracellular signal-regulated kinase pathways. Mol Cell Biol. 2006;26(6):2408-2418.

36. Ross EA, et al. Dominant suppression of inflammation via targeted mutation of the mRNA destabilizing protein tristetraprolin J Immunol. 2015;195(1):265-276.

37. Patial S, et al. Enhanced stability of tristetraprolin mRNA protects mice against immune-mediated inflammatory pathologies. Proc Natl Acad Sci U S A. 2016;113(7):1865-1870.

38. Ross EA, et al. Treatment of inflammatory arthritis via targeting of tristetraprolin, a master regulator of pro-inflammatory gene expression. Ann Rheum Dis. 2017;76(3):612-619.

39. Colin $\mathrm{S}$ et al. The antiangiogenic insulin receptor substrate-1 antisense oligonucleotide aganirsen impairs AU-rich mRNA stability by reducing 14-3-3 -tristetraprolin protein complex, reducing inflammation and psoriatic lesion size in patients. JPharmacol Exp Ther. 2014;349(1):107-117.

40. Grivennikov SI, et al. Distinct and nonredundant in vivo functions of TNF produced by t cells and macrophages/neutrophils: protective and deleterious effects. Immunity. 2005;22(1):93-104.

41. Brooks SA, Connolly JE, Diegel RJ, Fava RA, Rigby WF. Analysis of the function, expression, and subcellular distribution of human tristetraprolin. Arthritis Rheum. 2002;46(5):1362-1370.

42. Li L. Mouse epidermal keratinocyte culture. In: Randell SH, Fulcher ML, eds. Epithelial Cell Culture Protocols. 2nd ed. Totowa New Jersey: Humana Press; 2013:177-191.

43. Irizarry RA, et al. Exploration, normalization, and summaries of high density oligonucleotide array probe level data. Biostatistics. 2003;4(2):249-264.

44. Gautier L, Cope L, Bolstad BM, Irizarry RA. affy — analysis of Affymetrix GeneChip data at the probe level. Bioinformatics. 2004;20(3):307-15.

45. Ritchie ME, et al. limma powers differential expression analyses for RNA-sequencing and microarray studies. Nucleic Acids Res. 2015;43(7):e47.

46. Storey JD, Tibshirani R. Statistical significance for genomewide studies. Proc Natl Acad Sci U S A. 2003;100(16):9440-9445.

47. Chinetti-Gbaguidi G, et al. Human atherosclerotic plaque alternative macrophages display low cholesterol handling but high phagocytosis because of distinct activities of the PPAR $\gamma$ and LXR $\alpha$ pathways. Circ Res. 2011;108(8):985-995.

48. Silver JD, Ritchie ME, Smyth GK. Microarray background correction: maximum likelihood estimation for the normal-exponential convolution. Biostatistics. 2009;10(2):352-363.

49. Bolstad BM, Irizarry RA, Astrand M, Speed TP. A comparison of normalization methods for high density oligonucleotide array data based on variance and bias. Bioinformatics. 2003;19(2):185-193. 